\title{
HYPOELLIPTIC STOCHASTIC FITZHUGH-NAGUMO NEURONAL MODEL: MIXING, UP-CROSSING AND ESTIMATION OF THE SPIKE RATE
}

\author{
By José R. LEÓN ${ }^{*} \dagger, \ddagger, \S, 1$ AND Adeline SAMSON ${ }^{\dagger, 2}$ \\ Universidad de la República*, Université Grenoble Alpes ${ }^{\dagger}$, \\ INRIA project/team AIRSEA ${ }^{\ddagger}$ and Universidad Central de Venezuela ${ }^{\S}$ \\ The FitzHugh-Nagumo is a well-known neuronal model that describes \\ the generation of spikes at the intracellular level. We study a stochastic ver- \\ sion of the model from a probabilistic point of view. The hypoellipticity is \\ proved, as well as the existence and uniqueness of the stationary distribution. \\ The bi-dimensional stochastic process is $\beta$-mixing. The stationary density \\ can be estimated with an adaptive nonparametric estimator. Then we focus on \\ the distribution of the length between successive spikes. Spikes are difficult \\ to define directly from the continuous stochastic process. We study the distri- \\ bution of the number of up-crossings. We link it to the stationary distribution \\ and propose an estimator of its expectation. We finally prove mathematically \\ that the mean length of inter-up-crossings interval is equal to its up-crossings \\ rate. We illustrate the proposed estimators on a simulation study. Different \\ regimes are explored, with no, few or high generation of spikes.
}

1. Introduction. Neurons are excitable cells that are linked thanks to the synapses into a huge network. If the electric membrane potential, the voltage, of a neuron is sufficiently high, the neuron is able to produce an action potential, also called a spike, which is a stereotype fast and large electric signal. The spikes allow the neuron to activate its synaptic contacts and to modulate their voltage. The spikes can be viewed as the basic element of the information traveling from one neuron to another in the network. It is therefore of tremendous importance to understand and describe the individual voltage and the generation of spikes.

Neuronal spiking (also called firing) is a complex process that involves interactions between numerous cells. Modeling this mechanism mathematically is therefore difficult. Several neuronal models have been developed, the most famous is the 4 equations dynamical system of [12] that accurately describes the electrical mechanism of a single neuron. The model has an oscillatory behavior to reproduce

Received March 2017; revised September 2017.

${ }^{1}$ Supported in part by the INRIA International Chairs Program.

${ }^{2}$ Supported in part by the LabExPERSYVAL-Lab (ANR-11-LABX-0025-01).

MSC2010 subject classifications. Primary 60J70; secondary 62M05, 60H10, 60J60, 62P10, 35Q62, 37A50.

Key words and phrases. Hypoelliptic diffusion, FitzHugh-Nagumo model, invariant density, nonparametric estimation, up-crossings, pulse rate, spike rate estimation. 
the alternance of spiking phases and nonspiking phases. To produce such behavior, the differential equations driving the potential (denoted $X_{t}$ in the following) is coupled to differential equations related to the fraction of open ion channels of different kinds (conductances); however, this chaotic system is difficult to study from a mathematical point of view. Several relaxed models have then been proposed, most of them reducing the dimension of the system. We can cite the MorrisLecar model that simplifies the three channel equations of the Hodgkin-Huxley model into only one nonlinear equation modeling the membrane conductance evolution [20]. Another model is the FitzHugh-Nagumo (FHN) model, which has a polynomial drift. The FitzHugh-Nagumo and Morris-Lecar models share the properties of sub-threshold and supra-threshold response, that is, they intrinsically model the regenerative firing (spiking) mechanism in an excitable neuron. FitzHugh-Nagumo model is less plausible than conductance-based models: it has been built as an oscillatory system, not from physical assumptions. It has however the advantage of being more directly amenable to a mathematical analysis than the Hodgkin-Huxley or Morris-Lecar models thanks to its polynomial drift. Note that another class of neuronal models is the class of Leaky-Integrate-Fire (LIF) models (see [10], for a review), where the voltage is modeled by a one-dimensional process. Their main drawback is that spikes are not generated automatically and a (fixed) threshold has to be introduced, which is unrealistic.

Stochastic versions of neuronal models have been proposed to describe various sources of randomness [2, 10, 16]. The stochastic noise can be introduced in the first equation mimicking noisy presynaptic currents (see among others $[10,18,25])$. In the second class of stochastic models, the noise affects the other differential equations, describing the randomness of the conductance dynamics, like random opening and closing of ion channels [16, 17]. This second class of models can be viewed as a diffusion approximation of ion channels modeled by point processes (see among others [21]).

In this paper, we focus on the second class of stochastic FitzHugh-Nagumo (FHN) model. It is defined as follows. Let $X_{t}$ denote the membrane potential of the neuron at time $t$ and $C_{t}$ a recovery variable that models the channel kinetics. We assume that $\left(\left(X_{t}, C_{t}\right), t \geq 0\right)$ is governed by the following Itô stochastic differential equation (SDE):

$$
\left\{\begin{array}{l}
d X_{t}=\frac{1}{\varepsilon}\left(X_{t}-X_{t}^{3}-C_{t}-s\right) d t, \\
d C_{t}=\left(\gamma X_{t}-C_{t}+\beta\right) d t+\tilde{\sigma} d W_{t},
\end{array}\right.
$$

where $W_{t}$ is a standard Brownian motion, $\varepsilon$ is the time scale separation usually small ( $X$ has a faster time scale than $C$ ), $s$ is the magnitude of the stimulus current, $\tilde{\sigma}$ the diffusion coefficient, $\beta, \gamma$ are positive constants that determine the position of the fixed point and the duration of an excitation. FHN has already been studied extensively in physical papers (see, among others, $[2,16]$ ). Our objective is to revisit theoretically some of these results and to propose nonparametric estimators. 
The three objectives of the paper are the following. In Section 2, we study some probabilistic properties of the FitzHugh-Nagumo model: hypoellipticity, Feller, invariant probability, mixing property. To prove these results, we take advantage of the fact that a transformed version of the stochastic FitzHugh-Nagumo model with noise on the second equation is a generalization of van der Pol equations and belongs to the class of stochastic damping Hamiltonian systems. One of the main references is [26] for an overview of the theoretical properties of these models (see also [4, 13]). We first prove that the properties hold for the transformed stochastic damping Hamiltonian process and then establish the link between the two processes, thus showing the results also apply for the FitzHugh-Nagumo model.

In Section 3, we consider the questions related to the neuronal modeling. As said previously, spikes are the essential element of information exchange in the neural network. It is thus very important to understand their distribution. The distribution of spikes is difficult to study from scratch. Attempts have been proposed using point processes, but describe only the spike trains and not the neuronal voltage [22, 23]. When using voltage data, the first difficulty is the definition of the spike itself. In the pioneer work of Lindner and Schimansky-Geier [17], a spike is described as a "long" excursion on the phase space before returning back into the neighborhood of the fixed point. The pulse rate is measured by time averaging the number of pulses during time interval $[0, T]$ and the mean time between two pulses can also be estimated. They show that the pulse rate is the inverse of the mean length. But to our best knowledge, this has not be proven theoretically. Attempts have been based on Gaussian approximation of the voltage process [8, 25] leading to a Gaussian stochastic modeling. This Gaussian approximation does however not fit with real data. In this paper, we propose to study the spike generation through the modeling of the voltage by the FitzHugh-Nagumo model, avoiding any normal approximation or a vague definition of a spike. The idea is the following. A spike occurs when $X_{t}$ crosses a certain threshold, the spikes having then very similar shapes. Note that it is known from voltage data that the threshold is not fixed: the voltage $X_{t}$ has not always the same value when entering the spiking phases. We thus focus on the distribution of the process of up-crossings of $X_{t}$ at a large level $u$. If a spike occurs, the distribution of up-crossings should remain the same for any level value $u$ in a given interval. Finally, the distribution of the length of the interval between two successive spikes is studied and we prove that its expectation is the inverse of the up-crossing rate. Note that [3] also study the generation of spikes for a bi-dimensional FitzHugh-Nagumo model. Their model, although based on the same deterministic system as ours, has noise in both components. This fact implies that the solution $\left(X_{t}, C_{t}\right)$ is a classical diffusion (elliptic). Hence, each coordinate is a continuous but nondifferentiable function. The number of crossings of such a function is infinity in every bounded interval (allowing for instance the existence of local time). This prevents defining the spikes via the up-crossings. The authors define the spikes as large excursions in the space of phases and study their distributions. Their method is therefore very different from ours. 
In Section 4, we propose a nonparametric estimation of the stationary density based on a kernel estimator using only the discrete observations of the first coordinate $X_{t}$, following $[4,5]$. The two-dimensional bandwidth is selected automatically from the data with a Goldenshluger and Lepski's approach. We deduce an estimator of the up-crossing rate. These estimators are illustrated on simulations in different excitation regimes of the neuron. Note that the parameters of the system could be estimated by minimizing the contrasts proposed in [7, 24].

2. Properties of the FitzHugh-Nagumo model. Model (1.1) follows a nonlinear drift with singular diffusion coefficient (no noise on the first coordinate). It is not easy to study directly this kind of models because standard probabilistic tools assume a nondegenerate diffusion coefficient. To take advantage of probabilistic tools that have already been developed for some hypoelliptic systems, we introduce a change of variable of the second coordinate. This allows us to enter the class of stochastic damping Hamiltonian systems that have been widely studied (see among others $[4,13,26])$. We can then prove some useful properties for this model (hypoellipticity, Feller, existence of a stationary distribution, $\beta$-mixing) and prove them for the FitzHugh-Nagumo model as well. Let us first introduce the change of variable.

2.1. A stochastic damping Hamiltonian system. The change of variable is the following. Let $Y_{t}=\frac{1}{\varepsilon}\left(X_{t}-X_{t}^{3}-C_{t}-s\right)$. Applying Itô's formula, the FitzHughNagumo system (1.1) can be rewritten:

$$
\left\{\begin{array}{l}
d X_{t}=Y_{t} d t \\
d Y_{t}=\frac{1}{\varepsilon}\left(Y_{t}\left(1-\varepsilon-3 X_{t}^{2}\right)-X_{t}(\gamma-1)-X_{t}^{3}-(s+\beta)\right) d t-\frac{\tilde{\sigma}}{\varepsilon} d W_{t} .
\end{array}\right.
$$

Thanks to this transformation, we can notice that system (2.1) is a stochastic damping Hamiltonian system. These systems have been introduced to describe the dynamics of a particle with $X_{t}$ referring to its position and $Y_{t}$ to its velocity. The movement of the particle is guided by a potential $V(x)$ and by a damping force $c(x)$ :

$$
\left\{\begin{array}{l}
d X_{t}=Y_{t} d t \\
d Y_{t}=-\left(c\left(X_{t}\right) Y_{t}+\partial_{x} V\left(X_{t}\right)\right) d t+\sigma d W_{t}
\end{array}\right.
$$

Its infinitesimal generator $\mathcal{L}$ is

$$
\mathcal{L}=\frac{\sigma^{2}}{2} \partial_{y y}+y \partial_{x}-\left(c(x) y+\partial_{x} V(x)\right) \partial_{y} .
$$

These models have been studied by [26] under some conditions for $V(\cdot), c(\cdot)$ and the diffusion coefficient that we recall here:

(H1) The potential $V(x)$ is lower bounded, smooth over $\mathbb{R}, V$ and $\nabla V$ have polynomial growth at infinity. 
(H2) The damping coefficient $c(x)$ is continuous, for all $N>0$,

$$
\sup _{|x| \leq N}|c(x)|<+\infty
$$

and for all $x \in \mathbb{R}, c(x) \geq c$.

(H3) There exists $\sigma_{1}>0$ such that $0<\sigma<\sigma_{1}$.

In our case, the damping force is $c(x)=\frac{1}{\varepsilon}\left(3 x^{2}-1+\varepsilon\right)$, the potential is $V(x)=\frac{1}{\varepsilon}\left(\frac{x^{4}}{4}+\frac{\gamma-1}{2} x^{2}+(s+\beta) x\right)$ and the diffusion coefficient is $\sigma=\frac{\tilde{\sigma}}{\varepsilon}>0$. One can prove easily that conditions (H1), (H2) and (H3) are fulfilled under weak assumptions. Indeed:

- The potential $V(x)=\frac{1}{\varepsilon}\left(\frac{x^{4}}{4}+\frac{\gamma-1}{2} x^{2}+(s+\beta) x\right)$ is continuous, goes to $\infty$ when $x \rightarrow \pm \infty$ and is thus lower bounded, smooth over $\mathbb{R}, V$ and $\nabla V=\frac{1}{\varepsilon}\left(x^{3}+\right.$ $(\gamma-1) x+(s+\beta))$ have polynomial growth at infinity. This implies (H1).

- The damping coefficient $c(x)=\frac{1}{\varepsilon}\left(3 x^{2}-1+\varepsilon\right)$ is continuous, upper bounded on sets $\{|x| \leq N\}$ and for all $x \in \mathbb{R}, c:=1-\frac{1}{\varepsilon}$ implies (H2).

- (H3) is trivially verified since the diffusion coefficient is constant.

We can also notice that

$$
\frac{x \cdot \nabla V(x)}{|x|} \rightarrow+\infty \quad \text { as }|x| \rightarrow+\infty .
$$

This is condition (0.5) of [26]. It can be interpreted as follows: the force $-\nabla V(x)$ is "strong enough" for $|x|$ large to ensure a quick return of the system to compact subsets of $\mathbb{R}^{2}$.

2.2. Hypoellipticity and $\beta$-mixing of the transformed process. In this section, we prove some theoretical properties for the transformed process $\left(Z_{t}\right)=\left(X_{t}, Y_{t}\right)$ : $\left(Z_{t}\right)$ is strong Feller, hypoelliptic, the existence of a unique invariant probability and a $\beta$-mixing property. The main reference used in all the proofs is [26]. How these properties also hold for the original FitzHugh-Nagumo model is explained in Section 2.3.

Hypoellipticity and stationary distribution. We first focus on the hypoellipticity of $\left(Z_{t}\right)$.

Proposition 2.1 (Hypoellipticity). Let $Z_{t}=\left(X_{t}, Y_{t}\right)$ be the solution of system (2.1). The stochastic process $\left(Z_{t}\right)$ is hypoelliptic and strong Feller.

Hypoellipticity can be interpreted as the fact that the one-dimensional noise entering the second coordinate propagates to the two-dimensional space. It ensures that the distribution $P_{t}(z, \cdot)$ of the process $Z_{t}$ starting from $Z_{0}=z$ has a smooth density, denoted $p_{t}(z, \cdot)$ in the following. 
ProOF OF Proposition 2.1. We start by proving the hypoellipticity [19]. Let us denote $A_{0}, A_{1}$ the differential operators

$$
\begin{aligned}
& A_{0}=y \partial_{x}-(\nabla V(x)+c(x) y) \partial_{y}, \\
& A_{1}=\sigma \partial_{y} .
\end{aligned}
$$

Let $[A, B]$ denote the Lie bracket between operators $A$ and $B$. We have

$$
\left[A_{0}, A_{1}\right]=-\sigma \partial_{x}+\sigma c(x) \partial_{y} .
$$

Thus

$$
\operatorname{Span}\left(A_{0}, A_{1},\left[A_{0}, A_{1}\right]\right)=\operatorname{Span}\left(\partial_{x}, \partial_{y}\right) .
$$

This implies that the system is hypoelliptic.

Second, we prove that the stochastic process $\left(Z_{t}\right)$ is strong Feller. Let us denote $P_{t} f(z)=\mathbb{E}_{z}\left(f\left(Z_{t}\right)\right)=\int f(u) p_{t}(z, u) d u$ where $p_{t}(z, \cdot)$ is the transition density of the system. We want to prove that if $f$ is $L^{\infty}\left(\mathbb{R}^{2}\right)$ then $P_{t} f(x)$ is continuous. The coefficients of the infinitesimal generator $\mathcal{L}$ are $\mathcal{C}^{\infty}$. By Hörmander's theorem, this implies that $p_{t}(z, u)$ is $\mathcal{C}^{\infty}$. Thus as $f$ is bounded, $\int f(u) p_{t}(z, u) d u=P_{t} f(z)$ is continuous. So finally $\left(Z_{t}\right)$ is strong Feller.

We now prove the existence and uniqueness of an invariant probability. The main tool is to exhibit a Lyapounov function. We introduce the following function:

$$
\begin{aligned}
& \Psi(x, y)=e^{f(x, y)-\inf _{\mathbb{R}^{2}} f}, \\
& f(x, y)=a H(x, y)+b y G(x)+y W^{\prime}(x)+b U(x),
\end{aligned}
$$

where $H(x, y)=\frac{1}{2} y^{2}+V(x)$ is the Hamiltonian, the function $G(x)$ is such that

$$
G(x)=\Phi(|x|) \frac{x}{|x|} \quad \text { for } x \neq 0
$$

with $\Phi: \mathbb{R}^{+} \rightarrow \mathbb{R}^{+}$a nondecreasing smooth function equal to zero on a small neighborhood of 0 and equal to 1 for $|x| \geq 1$; the constant $a$ is such that $0<a<$ $\left(1-\frac{1}{\varepsilon}\right) \frac{1}{2 \sigma^{2}}$; the constant $b$ is such that

$$
b G^{\prime}(x)<\frac{1}{8}\left(a c-2 \sigma^{2} a^{2}\right)
$$

the function $W$ is of compact support, concave and such that for all $x$

$$
-W^{\prime \prime}(x) \geq-\frac{1}{8}\left(a c-2 \sigma^{2} a^{2}\right)
$$

and the function $U(x)$ is such that

$$
U^{\prime}(x)= \begin{cases}\frac{3}{\varepsilon} x^{2} \Phi(|x|) & \text { if } x \geq 0, \\ -\frac{3}{\varepsilon} x^{2} \Phi(|x|) & \text { if } x \leq 0 .\end{cases}
$$

We can prove the following proposition. 
Proposition 2.2 (Stationary distribution). Let $\left(X_{t}, Y_{t}\right)$ be the solution of system (2.1):

1. The function $\Psi(x, y)$ is a Lyapounov function: there exist a compact $K \in \mathbb{R}^{2}$ and constants $C, \xi>0$, such that

$$
-\frac{\mathcal{L} \Psi}{\Psi} \geq \xi 1_{K^{c}}-C 1_{K},
$$

and the Lyapounov function $\Psi$ is lower bounded by 1 .

2. The associated semi-group of the process $\left(X_{t}, Y_{t}\right)$ is strong Feller. All points of $\mathbb{R}^{2}$ are accessible. There exists a unique invariant probability measure $\mu a b$ solutely continuous with respect to the Lebesgue measure, with a smooth density denoted $p(\cdot): \mu(d z)=p(z) d z$.

3. Moments of any order of $\mu$ exist: for all $k_{1}, k_{2} \in \mathbb{N}$,

$$
\mathbb{E}\left(X_{t}^{k_{1}} Y_{t}^{k_{2}}\right)=\int x^{k_{1}} y^{k_{2}} d \mu(x, y)<+\infty .
$$

In the following, we will denote $p^{x}$ and $p^{y}$ the marginal of $p$ with respect to $x$ and $y$.

ProOF OF Proposition 2.2. 1. The choice of the Lyapounov function is not trivial. Following [26], we choose $\Psi(x, y)$ given by (2.3). With this form of $\Psi(x, y)$, we have

$$
-\frac{\mathcal{L} \Psi}{\Psi}=-\mathcal{L} f-\frac{1}{2} \sigma^{2}\left|\partial_{y} f\right|^{2} .
$$

Let us compute $-\frac{\mathcal{L} \Psi}{\Psi}$. We have

$$
\begin{aligned}
\mathcal{L} f= & \frac{a}{2} \sigma^{2}-a y^{2} c(x)+y^{2}\left(b G^{\prime}(x)+W^{\prime \prime}(x)\right) \\
& -y\left(b c(x) G(x)-b U^{\prime}(x)+c(x) W^{\prime}(x)\right) \\
& -V^{\prime}(x)\left(b G(x)+W^{\prime}(x)\right), \\
\frac{1}{2} \sigma^{2}\left(\partial_{y} f(x, y)\right)^{2}= & \frac{1}{2} \sigma^{2}\left(a y+b G(x)+W^{\prime}(x)\right)^{2} .
\end{aligned}
$$

We can bound $\frac{1}{2} \sigma^{2}\left(\partial_{y} f(x, y)\right)^{2}$ by

$$
\frac{1}{2} \sigma^{2}\left(\partial_{y} f(x, y)\right)^{2} \leq 2 \sigma^{2}\left(a^{2} y^{2}+\left(b G(x)+W^{\prime}(x)\right)^{2}\right) .
$$

Thus

$$
\begin{aligned}
-\frac{\mathcal{L} \Psi}{\Psi} \geq & -\frac{a}{2} \sigma^{2}+y^{2}\left(a c(x)-2 \sigma^{2} a^{2}-2\left(b G^{\prime}(x)+W^{\prime \prime}(x)\right)\right) \\
& +y\left(b\left(c(x) G(x)-U^{\prime}(x)\right)+c(x) W^{\prime}(x)\right) \\
& +V^{\prime}(x)\left(b G(x)+W^{\prime}(x)\right)-2 \sigma^{2}\left(b G(x)+W^{\prime}(x)\right)^{2} .
\end{aligned}
$$


Our choice of functions $G(x), U(x), W(x)$ and constants $a, b$ allows us to control the term in $y^{2}$ :

$$
\left(a c(x)-2 \sigma^{2} a^{2}-2\left(b G^{\prime}(x)+W^{\prime \prime}(x)\right)\right) \geq\left(a c-2 \sigma^{2} a^{2}-\frac{1}{2}\left(a c-2 \sigma^{2} a^{2}\right)\right)>0 .
$$

Now, we bound the term in $y$. First, note that by definition of $W$, there exists a constant $M_{1}$ such that

$$
y c(x) W^{\prime}(x) \geq-M_{1}|y|
$$

The funtion $U(x)$ verifies

$$
\sup _{x \in \mathbb{R}}\left|c(x) G(x)-U^{\prime}(x)\right|<+\infty .
$$

Thus, we obtain that there exists a constant $M_{2}$ such that

$$
y\left(b\left(c(x) G(x)-U^{\prime}(x)\right)+c(x) W^{\prime}(x)\right) \geq-M_{2}|y| .
$$

Now, we want to control the constant term $\left(b G(x)+W^{\prime}(x)\right)\left(V^{\prime}(x)-2 \sigma^{2} \times\right.$ $\left.\left(b G(x)+W^{\prime}(x)\right)\right)$. Given the form of function $G$ and $V^{\prime}(x)=x^{3}+x(\gamma-1)+$ $(s+\beta)$, we have that $V^{\prime}(x) G(x) \rightarrow+\infty$ as $|x| \rightarrow+\infty$ and that $G^{2}(x) \rightarrow+\infty$ as $|x| \rightarrow+\infty$. The function $W$ has compact support thus $W^{\prime}(x) V^{\prime}(x) \rightarrow 0$, $W^{\prime}(x) G(x) \rightarrow 0$ and $W^{\prime 2}(x) \rightarrow 0$ as $|x| \rightarrow+\infty$. So, the constant term is lower bounded.

Finally,

$$
\lim _{|x|+|y| \rightarrow \infty}\left(-\frac{\mathcal{L} \Psi}{\Psi}\right)=+\infty .
$$

We thus have the existence of a compact such that (2.4) holds.

Then we want to show that $\Psi$ is lower bounded by 1 . We can choose for any fixed $\delta>0$ the constant $a$ in $] \frac{c}{2 \sigma^{2}}-\frac{\delta}{2}, \frac{c}{2 \sigma^{2}}$ [. The Lyapounov function is defined as $\Psi(x, y)=e^{a H(x, y)+b y G(x)+y W^{\prime}(x)+b U(x)-\inf _{\mathbb{R}^{2}} F}$, we can prove that there exists a constant $B$ such that

$$
\Psi \geq B \exp \left(\left(\frac{c}{2 \sigma^{2}}-\delta\right) H(x, y)\right)
$$

Therefore, $\Psi \geq 1$.

2. We want to prove that there exists a unique invariant distribution.

First, note that the coefficients of our transformed model are $C^{\infty}\left(\mathbb{R}^{2}, \mathbb{R}\right)$. The hypoellipticity implies that the transition density $p_{t}\left(z, z^{\prime}\right)$ belongs to $C^{\infty}\left(\mathbb{R}^{2}, \mathbb{R}^{2}\right)$ $[4,26]$. Thus the semi-group is strong Feller.

By using the Girsanov formula shown in [26] (Lemma 1.1), the density satisfies $p_{t}\left(z, z^{\prime}\right)>0$ a.s. with respect to the Lebesgue measure $d z^{\prime}$ (see also [26]'s proof of the Proposition 1.2). Let $z \in \mathbb{R}^{2}$ and let $U$ be an open neighborhood of $z$. We define the resolvent operator $\mathcal{R}_{\lambda}$ as

$$
\mathcal{R}_{\lambda}(z, U)=\lambda \int_{0}^{\infty} e^{-\lambda t} P_{t}(z, U) d t
$$


where $P_{t}(z, U)=P_{t}^{z}\left(\mathbf{1}_{U}\right)=\int_{U} p_{t}\left(z, z^{\prime}\right) d z^{\prime}$. Using the result mentioned above, it holds that $P_{t}(z, U)>0$ for all $z \in \mathbb{R}^{2}$. Then for all $z$ we have that $\mathcal{R}_{\lambda}(z, U)>0$; hence, every point of $\mathbb{R}^{2}$ is accessible.

These two properties (strong Feller and every point accessible) give that the semi-group is strong Feller and that there exists a unique invariant measure. This measure is solution in distribution of the equation $\mathcal{L}^{*} \mu=0$. Using the same criterium of hypoellipticity, we deduce that this distribution is a $C^{\infty}\left(\mathbb{R}^{2}\right)$ function. Moreover, the semi-group is aperiodic because for any $K \subset \mathbb{R}^{2}$ compact we have $P_{t}(\cdot, K)>0$.

3. Applying Theorem 2.4 of [26] leads to the fact that $\mu$ satisfies $\int \Psi d \mu<+\infty$. Given the exponential form of $\Psi$, this means that $\mu$ integrates any polynomial in $(x, y)$, and thus the existence of any moments.

Mixing. We now study the mixing property of the stationary distribution. We first recall the definition.

DEFINITION 2.1. Let $\left\{Z_{t}\right\}_{t \in \mathbb{R}^{+}}$be a stationary stochastic process. Introducing the $\sigma$-algebra $\mathcal{F}_{t}=\sigma\left(\left\{Z_{s}: s \leq t\right\}\right)$ and $\mathcal{F}^{t}=\sigma\left\{Z_{s}: s \geq t\right\}$. We say that $Z$ is $\beta$ mixing, with mixing coefficient $\beta_{t}$, if

$$
\beta_{t}=\mathbb{E}\left[\sup \left\{\left|\mathbb{P}\left(U \mid \mathcal{F}_{u}\right)-\mathbb{P}(U)\right|: U \in \mathcal{F}^{u+t}\right\}\right]
$$

and $\beta_{t} \rightarrow 0$ when $t \rightarrow \infty$.

We can prove that the process $Z$ is $\beta$-mixing. Let us denote $B_{\Psi}$ the space of measurable functions

$$
B_{\Psi}=\left\{f: \mathbb{R}^{2} \rightarrow \mathbb{R}: \sup _{(x, y)} \frac{|f(x, y)|}{\Psi(x, y)}<\infty\right\},
$$

where $\Psi$ is a Lyapounov function defined by (2.3).

Proposition 2.3 (Mixing). Let $\left(X_{t}, Y_{t}\right)$ be the solution of system (2.1):

1. There exist constants $D>0$ and $0<\rho<1$ such that for all $z$, for any function $f \in B_{\Psi}$ :

$$
\left|P_{t} f(z)-\int f d \mu\right| \leq D \sup _{a}\left(\frac{\left|f(a)-\int f d \mu\right|}{\Psi(a)}\right) \Psi(z) \rho^{t} .
$$

2. The skeleton chain $\tilde{Z}_{k}=Z_{k h}$ for $h>0$ is exponentially $\beta$-mixing with $\beta$ mixing coefficient $\beta_{k h}$ such that

$$
\beta_{k h} \leq D^{\prime \prime}\|\Psi\|_{1} \rho^{k h} .
$$


PROOF. Let us start with the proof of 1 . Theorem 2.4 of [26] shows the existence of constants $D>0$ and $0<\rho<1$ such that for all $z$, inequality (2.5) holds. Then, since the Lyapounov function $\Psi$ is $\mu$ integrable and larger than 1, inequality (2.5) implies that the Markov chain $\left(Z_{i}\right)_{i \in \mathbb{N}}, Z_{0} \sim p_{s}(z) d z$ is exponentially $\beta$-mixing.

Now we prove 2. First, we remark that the Lyapounov function $\Psi$ is integrable with respect to the invariant measure (see [26]). Property 1 of Proposition 2.3 implies that

$$
\left\|P_{t}(f)-\int f d \mu\right\|_{1} \leq \rho^{t} D \sup _{a}\left(\frac{\left|f(a)-\int f d \mu\right|}{\Psi(a)}\right)\|\Psi\|_{1},
$$

where $\|\cdot\|_{1}$ denotes the $\mathbb{L}^{1}$ norm with respect to the invariant measure. We can deduce the following inequality in norm of total variation:

$$
\left\|P_{t}(z, \cdot)-\mu\right\|_{\mathrm{TV}} \leq D^{\prime \prime} \Psi(z) \rho^{t} .
$$

One can apply this inequality to the skeleton chain $\tilde{Z}_{k}=Z_{k h}$ for a certain $h$. Let us denote $\tilde{P}_{k}$ the discrete semi-group associated to $\tilde{Z}$. Then we get

$$
\left\|\tilde{P}_{k}(z, \cdot)-\mu\right\|_{\mathrm{TV}} \leq D^{\prime \prime} \Psi(z) \rho^{k h} .
$$

We can deduce from [9], Chapter 2, Section 2.4, that the $\beta$-mixing coefficient $\beta_{k h}$ is equal to

$$
\beta_{k h}=\int\left\|\tilde{P}_{k}(z, \cdot)-\mu\right\|_{\mathrm{TV}} d \mu(z) \leq D^{\prime \prime}\|\Psi\|_{1} \rho^{k h} .
$$

So we have $\beta_{k h} \leq D^{\prime \prime}\|\Psi\|_{1} \rho^{k h}$. Hence, the skeleton chain is exponentially $\beta$ mixing.

2.3. Stationary distribution and $\beta$-mixing of the FitzHugh-Nagumo process. The previous properties (existence and unicity of the stationary distribution, strong Feller, $\beta$-mixing) are proved for the transformed system (2.1). We now want to prove them for the original FitzHugh-Nagumo process (1.1). For that purpose, we establish the relation between the two systems. The function on the phase space relating the two processes is $F: \mathbb{R}^{2} \rightarrow \mathbb{R}^{2}$ defined as

$$
F:(x, c) \rightarrow(x, y), \quad F(x, c)=\left(x, \frac{1}{\varepsilon}\left(x-x^{3}-c-s\right)\right) .
$$

This function allows to prove the existence of a stationary distribution.

PROPOSITION 2.4 (Stationary distribution of the FitzHugh-Nagumo process). Let $\left(X_{t}, C_{t}\right)$ be the solution of system (1.1): $\mathbb{R}^{4}$

1. The transition density, denoted $p_{t}^{F}\left(x, c, x^{\prime}, c^{\prime}\right)$ verifies, for any $\left(x, c, x^{\prime}, c^{\prime}\right) \in$

$$
p_{t}^{F}\left(x, c, x^{\prime}, c^{\prime}\right)=\frac{1}{\varepsilon} p_{t}\left(x, c, F\left(x^{\prime}, c^{\prime}\right)\right)
$$


2. The associated semi-group of the process $\left(X_{t}, C_{t}\right)$ is strong Feller.

3. There exists a unique invariant probability measure $\mu^{F}$ with a smooth density denoted $p^{F}(\cdot)$ such that for any $(x, c) \in \mathbb{R}^{2}$

$$
p^{F}(x, c)=\frac{1}{\varepsilon} p\left(x, \frac{1}{\varepsilon}\left(x-x^{3}-c-s\right)\right)=\frac{1}{\varepsilon} p(F(x, c)),
$$

where $p(\cdot)$ is the density of the unique stationary distribution of system (2.1).

ProOF. First, let us compute the differential of the function $F$ :

$$
d F(x, c)=\left(\begin{array}{cc}
1 & 0 \\
\frac{1}{\varepsilon}\left(1-3 x^{2}\right) & -\frac{1}{\varepsilon}
\end{array}\right)
$$

Thus, its Jacobian is $J F(x, c)=\frac{1}{\varepsilon} \neq 0$.

1. We have for any set $A \in \mathbb{R}^{2}$, for any $(x, c) \in \mathbb{R}^{2}$,

$$
\begin{aligned}
\mathbb{E}^{(x, c)}\left[\mathbf{1}_{A}\left(X_{t}, C_{t}\right)\right] & :=\int_{A} p_{t}^{F}\left(x, c, x^{\prime}, c^{\prime}\right) d x^{\prime} d c^{\prime}=\mathbb{E}^{(x, c)}\left[\mathbf{1}_{F(A)}\left(X_{t}, Y_{t}\right)\right] \\
& =\int_{F(A)} p_{t}\left(x, c, x^{\prime}, y^{\prime}\right) d x^{\prime} d y \\
& =\frac{1}{\varepsilon} \int_{A} p_{t}\left(x, c, F\left(x^{\prime}, c^{\prime}\right)\right) d x^{\prime} d c^{\prime}
\end{aligned}
$$

Thus, we obtain

$$
p_{t}^{F}\left(x, c, x^{\prime}, c^{\prime}\right)=\frac{1}{\varepsilon} p_{t}\left(x, c, F\left(x^{\prime}, c^{\prime}\right)\right) .
$$

2. We define the semi-group associated to the process $\left(X_{t}, C_{t}\right)$ as follows. For any $(x, c) \in \mathbb{R}^{2}$, and for any bounded measurable function $f$, we set

$$
\begin{aligned}
P_{t}^{F}(f)(x, c) & =\int_{\mathbb{R}^{2}} f\left(x^{\prime}, c^{\prime}\right) p_{t}^{F}\left(x, c, x^{\prime}, c^{\prime}\right) d x^{\prime} d c^{\prime} \\
& =\frac{1}{\varepsilon} \int_{\mathbb{R}^{2}} f\left(x^{\prime}, c^{\prime}\right) p_{t}\left(x, c, F\left(x^{\prime}, c^{\prime}\right)\right) d x^{\prime} d c^{\prime} \\
& =\frac{1}{\varepsilon} \int_{\mathbb{R}^{2}} f\left(F^{-1}\left(x^{\prime}, y^{\prime}\right)\right) p_{t}\left(x, c, x^{\prime}, y^{\prime}\right) \frac{1}{J F\left(x^{\prime}, y^{\prime}\right)} d x^{\prime} d y^{\prime} \\
& =\mathbb{E}^{x, c}\left[f \circ F^{-1}\left(X_{t}, Y_{t}\right)\right]=P_{t}\left[f \circ F^{-1}\right](x, c) .
\end{aligned}
$$

As the transformed process $\left(X_{t}, Y_{t}\right)$ is strong Feller, we can deduce that for any bounded measurable function $f$, the term $P_{t}\left[f \circ F^{-1}\right](x, c)$ is a continuous function. Hence, the original process $\left(X_{t}, C_{t}\right)$ is strong Feller. 
3. For any function $f$ continuous and bounded, the sequence $\left\{P_{t}^{F}(\cdot) \mid t>0\right\}$ defined above is tight. Using the strong Feller property, the Krylov-Bogolioubov theorem yields that there exists at least one invariant probability distribution. Now, note that for any open set $U$, the strong Feller property implies that $P_{t}^{F}(U)$ is strictly positive. This implies the unicity of the invariant distribution.

Let us now prove (2.6). For any function $f$ continuous and bounded, we have by the ergodic theorem

$$
\begin{aligned}
\frac{1}{t} \int_{0}^{t} f\left(X_{s}, C_{s}\right) d s & =\frac{1}{t} \int_{0}^{t} f\left(F^{-1}\left(X_{s}, Y_{s}\right)\right) d s \\
& \rightarrow \int_{\mathbb{R}^{2}} f\left(F^{-1}(x, y)\right) p(x, y) d x d y .
\end{aligned}
$$

Setting $F^{-1}(x, y)=(x, c)$ it holds

$$
\int_{\mathbb{R}^{2}} f\left(F^{-1}(x, y)\right) p(x, y) d x d y=\frac{1}{\varepsilon} \int_{\mathbb{R}^{2}} f(x, c) p\left(x, \frac{1}{\varepsilon}\left(x-x^{3}-c-s\right)\right) d x d c .
$$

Hence, we obtain (2.6).

Now, we can prove that the process $\left(X_{t}, C_{t}\right)$ is also $\beta$-mixing.

Proposition 2.5 (Mixing of the FitzHugh-Nagumo process). The solution $\left(X_{t}, C_{t}\right)$ of the system (1.1) is $\beta$-mixing.

ProOF. Let us recall the link between the two processes, solutions of the systems (1.1) and (2.1):

$$
\left(X_{t}, C_{t}\right)=F^{-1}\left(X_{t}, Y_{t}\right) .
$$

The function $F$ is smooth. We can directly deduce that the property of $\beta$-mixing remains under this very simple transformation.

3. Number of up-crossings and spike rate. When the voltage is high enough, the emission of a spike occurs with probability 1 . This (high) level of up-crossing is not unique and fixed and this implies a difficulty to define properly a spike. In the following, we discuss and recall what have been described as spikes in the literature and then give a tentative definition through up-crossings.

\subsection{Spikes and previous results.}

Spiking regime. In the literature, spikes of the FitzHugh-Nagumo model have been defined as a long excursion in the phase space. One of the main references is [17]. An example of a phase space is given in Figure 1 for three different sets of parameters: left: $\varepsilon=0.1$, middle: $\varepsilon=0.4$, right: $\varepsilon=0.5$ (the other parameters 

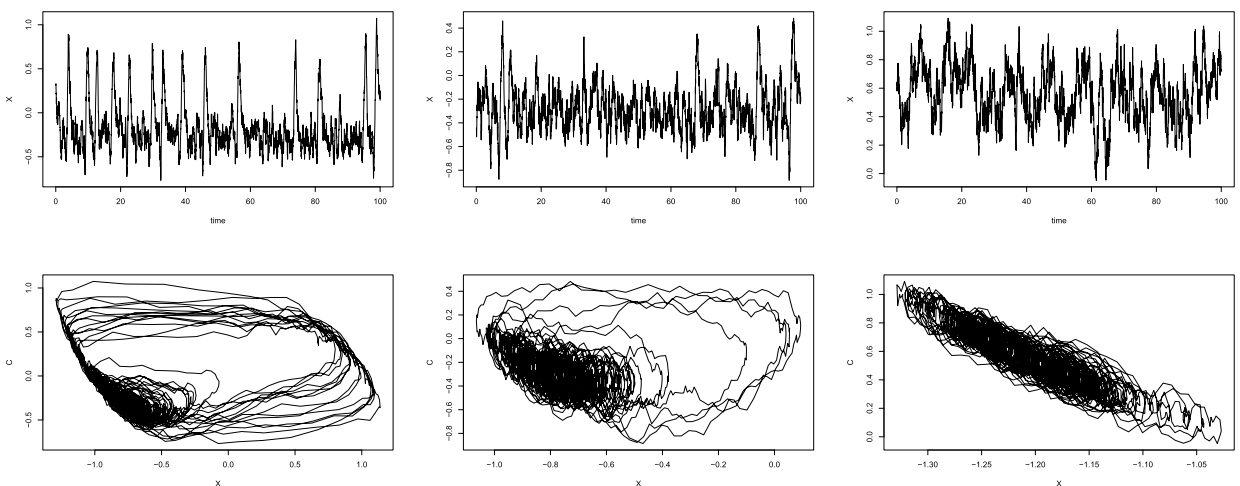

FIG. 1. Simulations of the FitzHugh-Nagumo. Voltage variable $X_{t}$ versus time (top line) and the corresponding trajectory in phase space $C_{t}$ versus $X_{t}$ (bottom line) for $s=0, \beta=0.8, \tilde{\sigma}=0.3$, left: $\varepsilon=0.1$ and $\gamma=1.5$, middle: $\varepsilon=0.4$ and $\gamma=1.5$, right: $\varepsilon=0.5$ and $\gamma=0.2$.

values are given in the description of the figure). Let us comment first the left plots $(\varepsilon=0.1)$ that generate automatically some spikes. As explained in [17], the fixed point is on the left bottom of the phase space. It corresponds to the dynamic of the potential between two pulses. Then the trajectory reaches the right branch, that belongs to the excited state: $X$ increases while $C$ remains almost constant. Then it moves along this branch upwards until it reaches its top, with $C$ that increases. Then it switches to the left branch that belongs to a refractory phase of the neuron: $X$ decreases and $C$ stays high. Finally, the trajectory relaxes into the fixed point with $X$ back to the resting potential and $C$ which decreases. When such a long excursion occurs, a "spike" or a "pulse" is observed in the voltage variable.

Now, let us comment the right plots of Figure 1 with $\varepsilon=0.5$. In that case, the potential stays in the vicinity of the fixed point and no excursion in the excited state occurs. Finally, let us comment the middle plots of Figure 1 with $\varepsilon=0.4$. We can observe excursions on the right branch, as when $\varepsilon=0.1$, but these excursions are less large. It is less clear if one should consider these excursions as spikes or pulses. The definition of a spike is therefore not clear.

Spike rate. Nevertheless, given this definition of a spike, the spike rate has been studied. We recall some results provided by Lindner and Schimansky-Geier [17]. Let $N_{t}$ denote the number of pulses during time interval $[0, t]$. The spike rate is defined as

$$
\rho:=\lim _{t \rightarrow \infty} \frac{N_{t}}{t}
$$

The process $N_{t}$ is random and the limit above is to be understood almost surely. Let us denote $T_{i}$ the $i$ th interspike interval, that is, the time between the $i$ and $i+1$ spikes (or pulses, or long excursions). The mean time $\langle T\rangle$ between two spikes, 
that is, the mean length of interspike intervals, can be defined using the ergodic theorem as

$$
\langle T\rangle=\lim _{N \rightarrow \infty} \frac{1}{N} \sum_{i=1}^{N} T_{i} .
$$

The spiking times $\left(T_{i}\right)$ are also random and the above limit is in the almost sure sense. Lindner and Schimansky-Geier [17] state the following relationship between these two quantities:

$$
\rho=\frac{1}{\langle T\rangle} .
$$

Relation (3.3) means that the limit in $t$ is equal to the limit in $N$ of the random processes $\left(N_{t}, t \geq 0\right)$ and $\left(T_{i}, i \geq 1\right)$. This relation is true for a Poisson process. It is however not straightforward for any point process. Moreover, the two processes $\left(N_{t}, t \geq 0\right)$ and $\left(T_{i}, i \geq 1\right)$ are difficult to define from the stochastic process $\left(X_{t}, C_{t}\right)$ or $\left(X_{t}, Y_{t}\right)$, and consequently it is difficult to prove (3.3).

The objective of the next two subsections is to give a formal mathematical framework to formula (3.1), (3.2) and (3.3). The starting point is a precise definition of the objects of study. The spikes are difficult to define from $\left(X_{t}, C_{t}\right)$ or $\left(X_{t}, Y_{t}\right)$ : should one take the beginning of the spike? the maximum?

An alternative is to study the number of spikes occurrence through the number of up-crossing of process $X_{t}$ at a certain (large) level $u$. This has the advantage of defining the occurrence of a spike through a precisely defined random variable (see below). Hence, theoretical results can be derived. This is the methodology we consider in this paper.

3.2. Number of up-crossings. The available neuronal data are the measurements of the membrane voltage $X_{t}$. We are interested in the process of upcrossings of this variable $X_{t}$. Note that this process can be defined through model (1.1) or through model (2.1). It is however more natural to use the transformed model (2.1). Indeed the process $Z_{t}=\left(X_{t}, Y_{t}\right)$ defines a measure $\mathbb{P}^{z}$ in the space $\Omega:=C\left(\mathbb{R}^{+}, \mathbb{R}^{2}\right)$. This means that $X_{t}$ is an a.s. continuously differentiable process and $\dot{X}_{t}=Y_{t}$. The number of up-crossings of process $X$. at level $u$ in $[0, t]$ is thus naturally defined as

$$
U_{t}^{X .}(u)=\#\left\{s \leq t: X_{s}=u, Y_{s}>0\right\}
$$

Heuristic of up-crossing process. Let us first give an intuition why $U_{t}^{X .}(u)$ is a process linked to the generation of spikes. If we forget the boundary effects, the random variable $N_{t}$ will be equal to the number of up-crossings $U_{t}^{X}$. $(u)$ at level $u$, for a set of large values $u$. Indeed, when a spike occurs, we expect $U_{t}^{X}(u)$ to be constant for all values $u$ that correspond to the right branch of the phase 
space (Figure 1), that is, to the (deterministic) increase phase of the potential. Note that for too large values of $u$ (larger than the maximum of the spikes), $U_{t}^{X}$. $(u)$ is expected to be zero. We therefore expect the distribution of $U_{t}^{X}$. to be stable for an interval of values of $u$ and then to decrease abruptly to 0 . On the contrary, if a neuron is not in a spiking regime (when $\varepsilon$ is large, e.g., see right plots of Figure 1), (small) excursions do not correspond to spikes and up-crossings will vary with $u$. These two very different behaviors of $U_{t}^{X}$. imply that knowing how process $U_{t}^{X}$. $(u)$ varies with $u$ gives automatically a definition of a spike and the fact that the neuron is in a spiking regime or not. Process $U_{t}^{X}$. $(u)$ can therefore be seen as a properly defined stochastic process that describes the generation of spikes. Note that a spike occurs the first time the process $\left(X_{t}, Y_{t}\right)$ hits the half-line $\left\{X_{s}=u, Y_{s}>0\right\}$ of $\mathbb{R}^{2}$.

It is therefore important to study the number of up-crossings. In this section, we first prove the Rice's formula that links the expectation of $U_{t}^{X}$. $(u)$ with the stationary density of process $Z_{t}=\left(X_{t}, Y_{t}\right)$. Then we prove an ergodic theorem for $U_{t}^{X}(u)$ : the expected value of $U_{t}^{X}$. $(u)$ by unit of time converges to an integral with respect to the stationary density. This limit, which depends on $u$, will be used in Section 3.3 to estimate the spike rate.

Rice's formula. We consider the norm $\|f\|_{\Psi}=\sup _{(x, y)} \frac{|f(x, y)|}{\Psi(x, y)}$. We first prove the Rice's formula on the expectation of the number of up-crossings.

Proposition 3.1 (Rice's formula). Let $Z_{t}=\left(X_{t}, Y_{t}\right)$ be the stationary solution of the FitzHugh-Nagumo system. The Rice's formula holds true

$$
\mathbb{E} U_{t}^{X .}(u)=t \int_{0}^{\infty} y p(u, y) d y .
$$

PROOF. The first step is the proof of a technical lemma.

LEMMA 3.1. Let us define the function $G_{y_{1}}(x, y):=\left|y-y_{1}\right|$. Set $z=(x, y)$. The semi-group $\left\{P_{t}\right\}_{t \geq 0}$ satisfies

$$
P_{t} G_{y_{1}}(z) \rightarrow G_{y_{1}}(x, y) \quad \text { if } t \rightarrow 0
$$

uniformly in $x$.

The proof of Lemma 3.1 is given in the Appendix. Then, Rice's formula is proved using the following result from [1]'s book, that we recall now.

Let $\left\{X_{t}\right\}_{t \in \mathbb{R}^{+}}$be a stochastic process and $Y$ its derivative. Let us first introduce the Rice's formula. For any $u \in \mathbb{R}$ and for $I$ an interval of time, where the crossings are counting, the Rice's formula is

$$
E\left(U_{t}^{X}(u)\right)=\int_{I} d t \int_{0}^{\infty} y p_{X_{t}, Y_{t}}(u, y) d y .
$$

It holds true under the following conditions: 
(C1) Function $(t, x) \rightarrow p_{X_{t}}(x)$ is continuous for $t \in I, x$ in a neighborhood of $u$.

(C2) $(t, x, y) \rightarrow p_{X_{t}, Y_{t}}(x, y)$ is continuous for $t \in I$ and $x$ in a neighborhood of $u$ and $y \in \mathbb{R}$.

(C3) Let define $A_{1}\left(t_{1}, t_{2}, x\right)=\int_{\mathbb{R}^{2}}\left|y_{1}-y\right| p_{X_{t_{1}}, Y_{t_{1}}, Y_{t_{2}}}\left(x, y, y_{1}\right) d y d y_{1}$. The process satisfies condition A41 on page 76 of [1]'s book if $A_{1}\left(t_{1}, t_{2}, x\right)$ tends to zero as $t_{2}-t_{1} \rightarrow 0$ uniformly, for $t_{1}$ and $t_{2}$ in a compact and $x$ in a neighborhood of $u$.

We must now verify if conditions (C1), (C2), (C3) hold. Under the stationary regime, the two marginal densities, $p^{x}(x)$ and $p^{y}(y)$, of the invariant density $p(x, y)$ correspond to the density of $X_{s}$ and the density of $Y_{s}$, respectively. They are both $C^{\infty}$ functions. By stationarity, the density of $\left(X_{s}, Y_{s}\right)$ is $p(x, y)$. Then (C1)-(C2) hold true.

Consider the vector $\left(X_{0}, Y_{0}, X_{s}, Y_{s}\right)$. It has a density $p_{s}\left(x, y, x_{1}, y_{1}\right) p(x, y)$. To prove (C3), let us observe that for any $s>0$, thanks to the stationary regime:

$$
A_{1}(s, x):=A_{1}(0, s, x)=\int_{\mathbb{R}^{2}}\left|y_{1}-y\right|\left(\int_{\mathbb{R}} p_{s}\left(x, y, x_{1}, y_{1}\right) p(x, y) d x_{1}\right) d y d y_{1} .
$$

Then we can write

$$
A_{1}(s, x):=\int_{\mathbb{R}}\left(P_{s} G_{y}\right)(x, y) p(x, y) d y .
$$

Lemma 3.1 implies that $P_{t}\left(G_{\tilde{y}}\right)(x, y) \rightarrow G_{\tilde{y}}(x, y)$ uniformly in $x$, where $(x, y)$ is the point of depart of our process. In the particular case $\tilde{y}=y$, we get $P_{t}\left(G_{y}\right)(x, y) \rightarrow G_{y}(x, y)=0$. This yields, by using the bounded convergence theorem, that $A_{1}(s, x) \rightarrow 0$ uniformly in $x$ and the Rice's formula for the first moment of the number of up-crossings holds true.

Ergodic theorem. We now prove that the ergodic theorem can be applied.

THEOREM 3.1. Let $Z_{t}=\left(X_{t}, Y_{t}\right)$ be the stationary solution of the FitzHughNagumo system. For any $u \in \mathbb{R}$,

$$
\frac{U_{t}^{X}(u)}{t} \rightarrow \int_{0}^{\infty} y p(u, y) d y \quad \text { a.s. }
$$

This result gives the limit of the expected number of up-crossings by unit of time. The fact that it is an integral with respect to the invariant density allows us to estimate this quantity (see Section 4). This limit will also be used in the estimation of the mean length between two spikes, as explained below.

Proof of Theorem 3.1. We follow [6], Section 11.5. Proposition 2.2 states that the process $\left(X_{t}, Y_{t}\right)$ is exponentially ergodic. Let us define the two processes 
of the number of up-crossings in interval $(s, s+1]$ and in interval $(s-1, s]$, respectively:

$$
\begin{array}{ll}
\zeta_{1}(s)=U_{s+1}^{X .}(u)-U_{s}^{X .}(u):=U_{(s, s+1]}^{X .}(u), & s \geq 0, \\
\zeta_{2}(s)=U_{s}^{X .}(u)-U_{s-1}^{X .}(u):=U_{(s-1, s]}^{X .}(u), & s \geq 1 .
\end{array}
$$

These two processes are stationary. Let $\theta$. denote the shift operator for the stationary Markov process $\left(X_{t}, Y_{t}\right)$. It holds $\zeta_{1}(s)=\theta_{s} \circ \zeta_{1}(0)$ and $\zeta_{2}(s)=\theta_{s} \circ \zeta_{2}(1)$. The ergodic theorem and Rice's formula (3.4) assure that

$$
\frac{1}{t} \int_{0}^{t} \zeta_{1}(s) d s \rightarrow \int_{0}^{\infty} y p(u, y) d y \quad \text { a.s. }
$$

and similarly for $\zeta_{2}$. Let us prove the following chain of inequalities from [6], page 238:

$$
\int_{0}^{t-1} \zeta_{1}(s) d s \leq U_{t}^{X} \cdot(u) \leq \int_{0}^{t+1} \zeta_{2}(s) d s
$$

holds. Let us show the left inequality

$$
\begin{aligned}
\int_{0}^{t-1} U_{(s, s+1]}^{X .}(u) d s & =\int_{0}^{t-1}\left(U_{s+1}^{X .}(u)-U_{s}^{X \cdot}(u)\right) d s \\
& =\int_{1}^{t} U_{s}^{X \cdot}(u) d s-\int_{0}^{t-1} U_{s}^{X \cdot}(u) d s \\
& =\int_{t-1}^{t} U_{s}^{X \cdot}(u) d s-\int_{0}^{1} U_{s}^{X \cdot}(u) d s \leq U_{t}^{X .}(u),
\end{aligned}
$$

where we have used that function $U_{t}^{X}(u)$ is nondecreasing. The right inequality can be proved similarly. Gathering (3.6) and (3.7) implies the theorem.

3.3. Spike rate. Now we want to link the spike rate $\rho$ with the up-crossing process. We start by the intuition and some heuristic, and then we formalize this link.

Heuristic. As explained earlier, the up-crossing process gives a definition of a spike: if the up-crossing process is constant for any level $u$ in a (large) interval, a spike occurs. Then, for a given level $u$ in this interval, the number of up-crossing $U_{t}^{X} .(u)$ is equal to the number of spikes. This naturally gives an approximation of the variable $N_{t}$ introduced by Lindner and Schimansky-Geier [17]. Let us define $\lambda(u)$ the limit of the number of up-crossings at level $u$ :

$$
\lambda(u)=\lim _{t \rightarrow \infty} \frac{U_{t}^{X}(u)}{t} .
$$


The ergodic theorem (Theorem 3.1) gives an explicit formula of this limit:

$$
\lambda(u)=\int_{0}^{\infty} y p(u, y) d y .
$$

Finally, we can naturally link the spike rate obtained via the random variable $N_{t}$ (excursions rate) and the rate of up-crossings:

$$
\lambda(u) \approx \rho .
$$

Formal link. This heuristic reasoning may be led by a more formal way. We want to describe formally the time between successive up-crossings of level $u$. Time between successive up-crossings has been studied by [6] (Chapter 11).

The idea is the following. Let $C_{[0, t]}^{X}(u)$ denote the number of all crossings at level $u$ on the interval $[0, t]$. Let us define for all $k \in \mathbb{N}^{*}$ the set

$$
H_{k}(\tau, t)=\mathbb{P}\left\{U_{(-\tau, 0)}^{X .}(u) \geq 1, C_{(0, t)}^{X .}(u) \leq k\right\} .
$$

The function of interest for us is when $k=1$. Assume an up-crossing occurs at time $\tau=0$. Then the trajectory is over level $u$ just after $\tau$. If $C_{(0, t)}^{X \text {. }}(u)=1$, the crossing is a down-crossing and no up-crossing occurs in interval $[0, t]$. Thus $H_{1}(\tau, t)$ is the probability to have a up-crossing in the interval $(-\tau, 0)$ and no up-crossing in interval $[0, t]$.

To study the interval between two spikes or up-crossings, we are interested in defining a conditional probability. For that purpose, let us introduce the following probability:

$$
\omega(t):=\mathbb{P}\{\text { there exists at least one up-crossing in time inteval }[0, t]\} .
$$

We know by using the ergodic theorem that

$$
U_{[0, t]}^{X}(u)=t \lambda(u)+o(t) \quad \text { when } t \rightarrow \infty .
$$

As the process $X$ is continuous and differentiable, the stream of up-crossings, that is, the times when the process crosses the level $u$ with positive derivative, is stationary and regular. This implies that $\mathbb{P}\left\{U_{[0, t]}^{X .}(u)>1\right\}=o(t)$. From [6] (page 54), we can prove that

$$
\omega(t)=t \ell(u)+o(t) \quad \text { when } t \rightarrow 0
$$

for a certain constant $\ell(u)$. The property of a regular up-crossing process implies that $\ell(u)=\lambda(u)$. This important fact links the ergodic limit (3.10) with the behavior of $\omega(t)$ in a neighborhood of zero. This result will be very useful for us.

Then [6] proves that for all $k$, there exists a finite function $\Phi_{k}$ defined by the following limit:

$$
\Phi_{k}(t):=\lim _{\tau \rightarrow 0} \frac{H_{k}(\tau, t)}{\omega(\tau)}
$$


This last function represents the conditional probability of no more than $k$ crossings in the interval $(0, t)$, given that an up-crossing occurred "at" time zero. For $k=1, \Phi_{1}(t)$ is the conditional probability that no up-crossing occurs in interval $[0, t]$, given that an up-crossing occurred "at" time zero.

It is then natural to introduce the following function:

$$
F_{2}(t):=1-\Phi_{1}(t) .
$$

The first remarkable fact is that $F_{2}(\cdot)$ is a nondefective distribution function.

Proposition 3.2. The function $F_{2}$ defined by (3.12) is the cumulative distribution function of a probability measure.

Moreover, it may be regarded as the distribution function of the length of the interval of an arbitrarily chosen up-crossing and the next up-crossing, following [6].

Proof. It holds easily that $0 \leq F_{2}(t) \leq 1$.

Then to prove that it is a cumulative distribution function, set $u_{0}(t)=$ $\mathbb{P}\left\{U_{(0, t)}^{X .}=0\right\}$. We have

$$
\begin{aligned}
u_{0}(t)-u_{0}(t+\tau) & =\mathbb{P}\left\{U_{(0, t)}^{X .}(u)=0\right\}-\mathbb{P}\left\{U_{(-\tau, t)}^{X .}(u)=0\right\} \\
& =\mathbb{P}\left\{U_{(-\tau, t)}^{X .}(u) \geq 1, U_{(0, t)}^{X .}(u)=0\right\} \\
& =H_{1}(\tau, t)+o(\tau),
\end{aligned}
$$

where we use [6] (page 225) for the last equality: "the probability of more than one crossing in $(-\tau, 0)$ is $o(\tau)$, whereas if the only crossing in $(-\tau, 0)$ is an upcrossing, then $U^{X} \cdot(0, t)=0$ when (and only when) $C_{(0, t)}^{X .}(u)=0$ ”.

Thus

$$
\lim _{\tau^{+} \rightarrow 0} \frac{u_{0}(t+\tau)-u_{0}(t)}{\tau}=-\lim _{\tau \rightarrow 0} \frac{H_{1}(\tau, t)}{\omega(\tau)} \frac{\omega(\tau)}{\tau}=-\lambda(u) \Phi_{1}(t) .
$$

Thus the function $u_{0}$ has right-hand side derivative: $D^{+} u_{0}(t)=-\lambda(u) \Phi_{1}(t)$. Moreover, the Lebesgue theorem gives

$$
u_{0}(T)-u_{0}(0)=\int_{0}^{T} D^{+} u_{0}(t) d t .
$$

As $u_{0}$ is bounded, the derivative $D^{+} u_{0}(t)$ is integrable over $(0, \infty)$. This in particular implies that $D^{+} u_{0}(t) \rightarrow 0$ whenever $t \rightarrow \infty$, thus $\lim _{t \rightarrow \infty} \Phi_{1}(t)=0$ and, therefore, $F_{2}(t) \rightarrow 1$ when $t \rightarrow \infty$. Finally, as $F_{2}$ is nondecreasing it is a cumulative distribution function of a probability measure.

As $F_{2}$ can be interpreted as the distribution function of the length of an interup-crossings interval (interval between two successive up-crossings), we are interested in computing its first two moments. Following Cramér and Leadbetter ([6], page 227), we obtain the following. 
PROPOSITION 3.3. The expectation of the distribution $F_{2}$, that is, the mean length of the interval between two successive up-crossings, is given by

$$
\int_{0}^{\infty} t d F_{2}(t)=\frac{1}{\lambda(u)}
$$

The second moment of $F_{2}$ is

$$
\int_{0}^{\infty} t^{2} d F_{2}(t)=\frac{2}{\lambda(u)} \int_{0}^{\infty} u_{0}(t) d t
$$

where $u_{0}(t)=\mathbb{P}\left\{U_{(0, t)}^{X}=0\right\}$.

The expectation of the length of an inter-up-crossings interval is equal to the inverse of the rate of up-crossings at level $u$ [formula (3.9)], and thus to the spike rate. This is the principal advantage of our approach. We give a theoretical justification to the link between the length of the excursions and the spike rate.

Proof of Proposition 3.3. Start with the mean of $F_{2}$. Given formula (3.13), one easily obtains

$$
\int_{0}^{\infty} t d F_{2}(t)=\int_{0}^{\infty}\left[1-F_{2}(t)\right] d t=\frac{1}{\lambda(u)}\left[u_{0}(0)-u_{0}(\infty)\right] .
$$

Then we use that $u_{0}(0)=1$ and $u_{0}(\infty)=0$ to obtain

$$
\int_{0}^{\infty} t d F_{2}(t)=\frac{1}{\lambda(u)}
$$

For the second moment, we simply apply twice an integration by parts to obtain

$$
\int_{0}^{\infty} t^{2} d F_{2}(t)=\frac{2}{\lambda(u)} \int_{0}^{\infty} u_{0}(t) d t
$$

Finally, estimating $\lambda(u)$ gives a direct estimation of the mean length of interspike intervals, as well as its variance. This is detailed in Section 4.

4. Estimation of invariant density and spike rate. The objective is to estimate the spike rate and $\lambda(u)$. In the neuronal context, the coordinate $Y_{t}$ cannot be measured and only discrete observations of $X$ at discrete times $i \delta, i=1, \ldots, n$ with discretization step $\delta$ are available. It offers the possibility to work rather through model (1.1) or model (2.1). As shown in the previous section, the spike rate and $\lambda(u)$ are easier to define and estimate using model (2.1) and its stationary density $p$. We thus start with the invariant density. 
4.1. Invariant density estimation. The density $p$ has no explicit formula. We therefore use the nonparametric adaptive estimation of $p$ from observations $\left(X_{1 \delta}, \ldots, X_{n \delta}\right)$, proposed by Comte, Prieur and Samson [5].

Let us detail their estimator. Let $K$ be some kernel $\mathcal{C}^{2}$ function with compact support $A$ such that its partial derivatives functions $\frac{\partial K}{\partial x}$ and $\frac{\partial K}{\partial y}$ are in $\mathbb{L}^{2}(\mathbb{R})$, $\int K(x, y) d x d y=1$ and $\int K^{2}(x, y) d x d y<\infty$. For any bandwidth $b=\left(b_{1}, b_{2}\right)$ with $b_{1}>0, b_{2}>0$, for all $(x, y) \in \mathbb{R}^{2}$, we denote

$$
K_{b}(x, y)=\frac{1}{b_{1} b_{2}} K\left(\frac{x}{b_{1}}, \frac{y}{b_{2}}\right) .
$$

When both coordinates are observed, the natural estimator of $p$ for all $z=$ $(x, y) \in \mathbb{R}^{2}$ is

$$
\tilde{p}_{b}(z)=\tilde{p}_{b}(x, y):=\frac{1}{n} \sum_{i=1}^{n} K_{b}\left(x-X_{i \delta}, y-Y_{i \delta}\right)=\frac{1}{n} \sum_{i=1}^{n} K_{b}\left(z-Z_{i \delta}\right) .
$$

When only $X$ is observed, we replace $Y$ by the increments of $X$. Indeed, for any $i=1, \ldots, n$, when $\delta$ is small enough, we have

$$
X_{(i+1) \delta}-X_{i \delta}=\int_{i \delta}^{(i+1) \delta} Y_{t} d t \approx \delta Y_{i \delta}
$$

Let us thus define the following approximation of $Y_{i \delta}$ :

$$
\bar{Y}_{i \delta}=\frac{X_{(i+1) \delta}-X_{i \delta}}{\delta}
$$

and define the two-dimensional kernel estimator by

$$
\hat{p}_{b}(x, y):=\frac{1}{n} \sum_{i=1}^{n} K_{b}\left(x-X_{i \delta}, y-\bar{Y}_{i \delta}\right) .
$$

The bandwidth $b=\left(b_{1}, b_{2}\right)$ has to be chosen to realize a trade-off between the bias of $\hat{p}_{b}$ and its variance. This is automatically achieved using the adaptive estimation procedure proposed by [5]. We can apply their procedure because we have already proved that the invariant density $p$ decreases exponentially and is $\beta$-mixing (Section 2). Their procedure, inspired by [11], is the following. Let $\mathcal{B}_{n}=\left\{\left(b_{1, k}, b_{2, \ell}\right), k, \ell=1 / \sqrt{n}, \ldots, c / \sqrt{n}\right\}$ be the set of possible bandwidths. Set for all $z=(x, y)$ and all $b, b^{\prime} \in \mathcal{B}_{n}$

$$
\hat{p}_{b, b^{\prime}}(z)=K_{b^{\prime}} \star \hat{p}_{b}(z)=\frac{1}{n} \sum_{i=1}^{n} K_{b^{\prime}} \star K_{b}\left(x-X_{i \delta}, y-\bar{Y}_{i \delta}\right) .
$$

Now let

$$
A(b)=\sup _{b^{\prime} \in \mathcal{B}_{n}}\left(\left\|\hat{p}_{b, b^{\prime}}-\hat{p}_{b^{\prime}}\right\|^{2}-V\left(b^{\prime}\right)\right)_{+}
$$


with

$$
V(b)=\kappa_{1} \frac{1}{n b_{1} b_{2}} \sum_{i=0}^{n-1} \beta(i \delta)+\kappa_{2} \frac{\delta}{b_{1} b_{2}^{3}},
$$

where $\kappa_{1}, \kappa_{2}$ are numerical constants and $\beta(i \delta)$ are the $\beta$-mixing coefficients. The selection is then made by setting

$$
\hat{b}=\arg \min _{b \in \mathcal{B}_{n}}(A(b)+V(b)) .
$$

Comte, Prieur and Samson [5] prove an oracle inequality for the final estimator $\hat{p}_{\hat{b}}$.

THEOREM 4.1 ([5]). Set $p_{b}(z)=K_{b} \star p$ the function that is estimated without bias by $\hat{p}_{b}$. We have

$$
\mathbb{E}\left(\left\|\hat{p}_{\hat{b}}-p\right\|^{2}\right) \leq C \inf _{b \in \mathcal{B}_{n}}\left(\left\|p_{b}-p\right\|^{2}+V(b)\right)+C \frac{\log (n)}{n \delta} .
$$

As explained in [5], the Goldenshluger and Lepski's procedure (4.4) is numerically demanding due to the double convolutions $\hat{p}_{b, b^{\prime}}$, especially in the multidimensional case. They propose a simplified procedure inspired on the one proposed by [15] in the independent observation case, that we also implement in this paper. The selection of the bandwidth is the following:

$$
\hat{\hat{b}}=\arg \min _{b \in \mathcal{B}_{n}}\left(\left\|\hat{p}_{b}-\hat{p}_{b_{\min }}\right\|^{2}+V(b)\right)
$$

with $\kappa_{1}=0.1$ and $\kappa_{2}=0.001$ and $b_{\min }=(1 / \sqrt{n}, 1 / \sqrt{n})$, as given in [5]. By plugging $\hat{\hat{b}}$ into (4.3), we obtain $\hat{p}:=\hat{p}_{\hat{b}}$ which is the final estimator of $p$.

4.2. Spike rate estimation. Equation (3.8) provides a good start to estimate the spike rate. The quantity that we estimate is $\lambda(u)$ for a large level $u$. By plugging the kernel estimator $\hat{p}$ of the invariant density, we define the following estimator of $\lambda(u)$ :

$$
\hat{\lambda}(u)=\int_{0}^{\infty} y \hat{p}(u, y) d y .
$$

For some specific choices of kernel $K$, the estimator $\hat{\lambda}(u)$ has an explicit expression. More precisely, let us consider a multiplicative two-dimensional kernel $K(x, y)=k(x) k(y)$, where $k$ is a continuous and bounded kernel, such that $\int k(v) d v=1$. Then we have

$$
\hat{p}(u, y)=\frac{1}{n \hat{b}_{1} \hat{b}_{2}} \sum_{i=1}^{n} k\left(\frac{u-X_{i \delta}}{\hat{b}_{1}}\right) k\left(\frac{y-\bar{Y}_{i \delta}}{\hat{b}_{2}}\right),
$$


with $\hat{b}_{1}, \hat{b}_{2}$ the bandwidth estimated adaptively by (4.5). We get

$$
\begin{aligned}
\hat{\lambda}(u) & =\frac{1}{n \hat{b}_{1} \hat{b}_{2}} \sum_{i=1}^{n} k\left(\frac{u-X_{i \delta}}{\hat{b}_{1}}\right) \int_{0}^{\infty} y k\left(\frac{y-\bar{Y}_{i \delta}}{\hat{b}_{2}}\right) d y \\
& =\frac{1}{n \hat{b}_{1}} \sum_{i=1}^{n} k\left(\frac{u-X_{i \delta}}{\hat{b}_{1}}\right)\left(\hat{b}_{2} \int_{-\frac{\bar{Y}_{i \delta}}{\hat{b}_{2}}}^{\infty} y k(y) d y+\bar{Y}_{i \delta} \int_{-\frac{\bar{Y}_{i \delta}}{\hat{b}_{2}}}^{\infty} k(y) d y\right) .
\end{aligned}
$$

For a Gaussian centered kernel $k$, we obtain

$$
\hat{\lambda}(u)=\frac{1}{n \hat{b}_{1}} \sum_{i=1}^{n} k\left(\frac{u-X_{i \delta}}{\hat{b}_{1}}\right)\left(\frac{\hat{b}_{2}}{\sqrt{2 \pi}} e^{-\frac{1}{2}\left(\frac{\bar{Y}_{i \delta}}{\hat{b}_{2}}\right)^{2}}+\bar{Y}_{i \delta}\left(1-\Phi\left(-\frac{\bar{Y}_{i \delta}}{\hat{b}_{2}}\right)\right)\right),
$$

where $\Phi(\cdot)$ is the cumulative distribution function of the centered and reduced normal distribution.

The next step is the estimation of the variance, and more precisely of the second moment of $F_{2}$, given by (3.14). First, we need to estimate $u_{0}(t)=\mathbb{P}\left\{U_{(0, t)}^{X}=0\right\}$. The idea is to link this function with the survival function of the inter-up-crossings interval.

For a fixed level $\mathrm{u}$, let us assume that one up-crossing occurs at time 0 and let denote $\left\{T_{i}^{u}, i \geq 0\right\}$ the successive times of up-crossings after time 0 with $T_{0}^{u}=0$.

Thanks to the stationarity of the process, the $\left(T_{i+1}^{u}-T_{i}^{u}, i \geq 0\right)$ are identically distributed. For any $i \geq 0$, we can rewrite $u_{0}(t)$ as follows:

$$
u_{0}(t)=\mathbb{P}\left(U_{(0, t)}^{X}=0\right)=\mathbb{P}\left(T_{1}^{u}>t\right)=\mathbb{P}\left(\left(T_{i+1}^{u}-T_{i}^{u}\right)>t\right) .
$$

A natural estimator of $u_{0}(t)$ from observations on interval $[0, T]$ is

$$
\hat{u}_{0}^{T}(t)=\frac{1}{U_{(0, T)}^{X}} \sum_{i=0}^{U_{(0, T)}^{X}} \mathbf{1}_{\left(T_{i+1}^{u}-T_{i}^{u}\right)>t}
$$

where $U_{(0, T)}^{X}$ is the number of up-crossings in the interval $[0, T]$.

LEMMA 4.1. Estimator $\hat{u}_{0}^{T}(t)$ (4.7) based on observations on interval $[0, T]$ is a consistent estimator of $u_{0}(t)$ when $T$ goes to infinity.

ProOF. Set $[\cdot]$ for the integer part. Let us rewrite $\hat{u}_{0}^{T}(t)$ as

$$
\hat{u}_{0}^{T}(t)=\frac{1}{\frac{U_{(0, T)}^{X}}{T}} \frac{1}{T} \sum_{i=0}^{U_{(0, T)}^{X}} \mathbf{1}_{\left(T_{i+1}^{u}-T_{i}^{u}\right)>t} .
$$


By the ergodic theorem, we have $U_{(0, T)}^{X} \sim T \int_{0}^{\infty} y p(u, y) d y$ a.s. We can thus deduce that

$$
\begin{aligned}
& \left|\frac{1}{T}\left(\sum_{i=0}^{U_{(0, T)}^{X}} \mathbf{1}_{\left(T_{i+1}^{u}-T_{i}^{u}\right)>t}-\sum_{i=0}^{\left[T \int_{0}^{\infty} y p(u, y) d y\right]} \mathbf{1}_{\left(T_{i+1}^{u}-T_{i}^{u}\right)>t}\right)\right| \\
& \quad \leq\left|\frac{U_{(0, T)}^{X}}{T}-\frac{\left[T \int_{0}^{\infty} y p(u, y) d y\right]}{T}\right|
\end{aligned}
$$

Moreover,

$$
\lim _{T \rightarrow \infty}\left|\frac{U_{(0, T)}^{X}}{T}-\frac{\left[T \int_{0}^{\infty} y p(u, y) d y\right]}{T}\right|=0 .
$$

Thus we have $\lim _{T \rightarrow \infty} \hat{u}_{0}^{T}(t)=\lim _{T \rightarrow \infty} \frac{1}{\frac{U_{(0, T)}^{X}}{T}} \frac{1}{T} \sum_{i=0}^{\left[T \int_{0}^{\infty} y p(u, y) d y\right]} \mathbf{1}_{\left(T_{i+1}^{u}-T_{i}^{u}\right)>t}$.

We now focus on studying the RHS. The stationarity and the ergodic theorem imply that

$$
\frac{1}{T} \sum_{i=0}^{\left[T \int_{0}^{\infty} y p(u, y) d y\right]} \mathbf{1}_{\left(T_{i+1}^{u}-T_{i}^{u}\right)>t} \rightarrow \int_{0}^{\infty} y p(u, y) d y \mathbb{P}\left(T_{1}^{u}>t\right) \quad \text { a.s. }
$$

So finally we obtain

$$
\begin{aligned}
\lim _{T \rightarrow \infty} \hat{u}_{0}^{T}(t) & =\frac{1}{\int_{0}^{\infty} y p(u, y) d y} \int_{0}^{\infty} y p(u, y) d y \mathbb{P}\left(T_{1}^{u}>t\right) \\
& =\mathbb{P}\left(T_{1}^{u}>t\right)=u_{0}(t) \quad \text { a.s. }
\end{aligned}
$$

To estimate the second moment of $F_{2}$, we plug $\hat{u}_{0}^{T}(t)$ into formula (3.14):

$$
\frac{2}{\hat{\lambda}(u)} \int_{0}^{\infty} \hat{u}_{0}^{T}(t) d t=\frac{2}{\hat{\lambda}(u)} \frac{1}{U_{(0, T)}^{X}} \sum_{i=0}^{U_{(0, T)}^{X}}\left(T_{i+1}^{u}-T_{i}^{u}\right)=\frac{2}{\hat{\lambda}(u)} \frac{T_{U_{(0, T)}^{X}}^{u}}{U_{(0, T)}^{X}} .
$$

The final estimator of the variance of the length between two successive upcrossings at level $u$ based on observations on the interval $[0, T]$ is thus

$$
\hat{V}^{u}=\frac{2}{\hat{\lambda}(u)} \frac{T_{U_{(0, T)}^{X}}^{u}}{U_{(0, T)}^{X}}-\frac{1}{\hat{\lambda}(u)^{2}} .
$$

5. Simulation. Three sets of parameter values of the FitzHugh-Nagumo model are used in the simulations (same as above). A set that allows spike generation: $\varepsilon=0.1, s=0, \gamma=1.5, \beta=0.8, \sigma=0.3$; a set that generates small excursions $s=0, \beta=0.8, \tilde{\sigma}=0.3, \varepsilon=0.4$ and $\gamma=1.5$ and a set that does not 

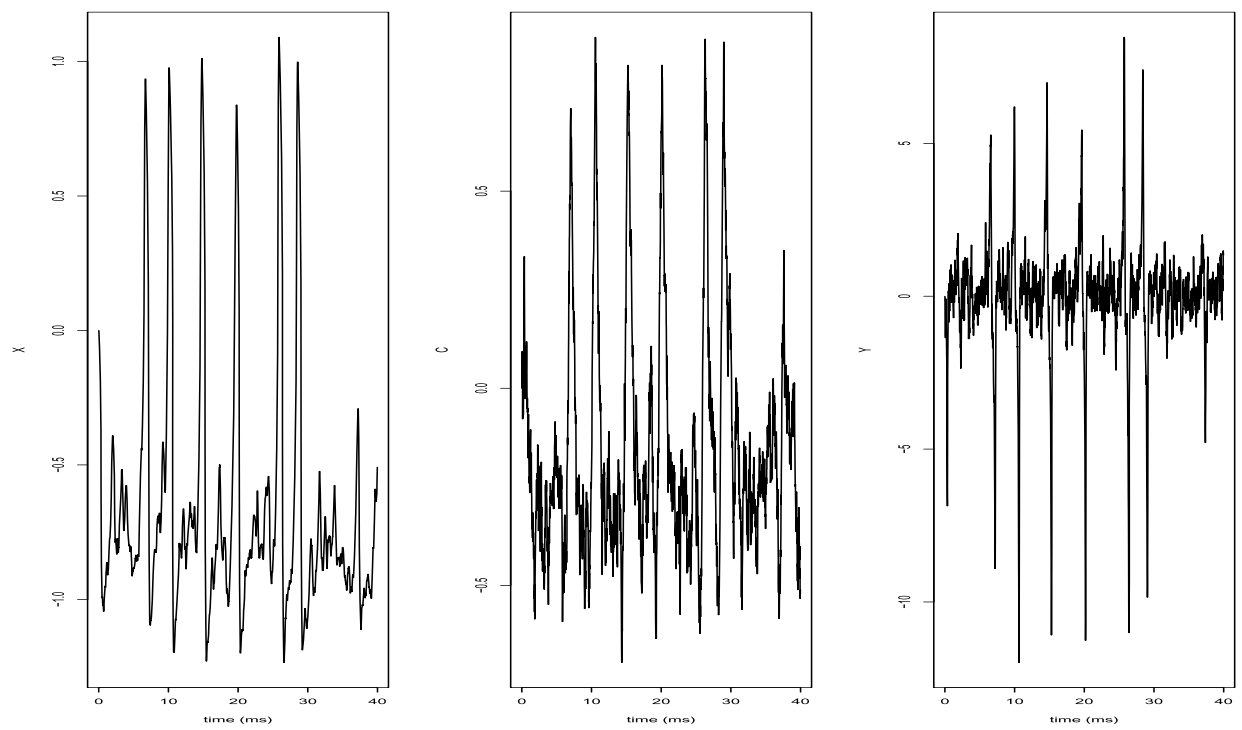

FIG. 2. FitzHugh-Nagumo simulation. Left: coordinate X along time. Middle: coordinate C. Right: transformed coordinate $Y$. Simulations are performed with $\delta=0.02, n=2000$. Parameters are $\varepsilon=0.1, s=0, \gamma=1.5, \beta=0.8, \sigma=0.3$.

generate spikes $s=0, \beta=0.8, \tilde{\sigma}=0.3, \varepsilon=0.5$ and $\gamma=0.2$. Trajectories are simulated with time step $\delta=0.02 \mathrm{~ms}, n=20,000$ and a Itô-Taylor scheme of order 2 of system (1.1). Figure 2 shows an example of such a simulation with trajectories of $\left(X_{t}, C_{t}\right)$ for the first set of parameters that generates spikes $(s=0$, $\beta=0.8, \tilde{\sigma}=0.3, \varepsilon=0.1$ and $\gamma=1.5)$, as well as the transformed coordinate $Y_{t}=\frac{1}{\varepsilon}\left(X_{t}-X_{t}^{3}-C_{t}-s\right)$ between 0 and $200 \mathrm{~ms}(n=1000)$.

We apply the adaptive estimation procedure (4.5) to estimate the invariant density $p$. The true density $p$ has no closed form. To compare the estimator with the truth, we approximate $p$ by numerically solving the associated hypoelliptic Fokker-Planck equation. A finite difference method is used to solve the FokkerPlanck equation [14]. The solver is very stable for the set of parameters that does not generate spikes $(\varepsilon=0.5)$. This is illustrated in the bottom plots of Figure 3 . The density estimator $\hat{p}$ (red dotted line) is very closed to the "true" stationary density $p$ (black plain line). However, the finite difference approximation of stationary density appears to be unstable for the set of parameters that generates spikes or even small excursions (see black lines of top and middle plots of Figure 3). We tried to decrease the step of the discretization grid but the approximation remains unstable (recall that the PDE is hypoelliptic). We then compare the estimator with a Monte Carlo approximation of the stationary density. More precisely, 10,000 trajectories have been simulated on an interval $[0, T=n \delta]$. The last point of each trajectory is stored in a sample of i.i.d. simulation under the stationary regime. 

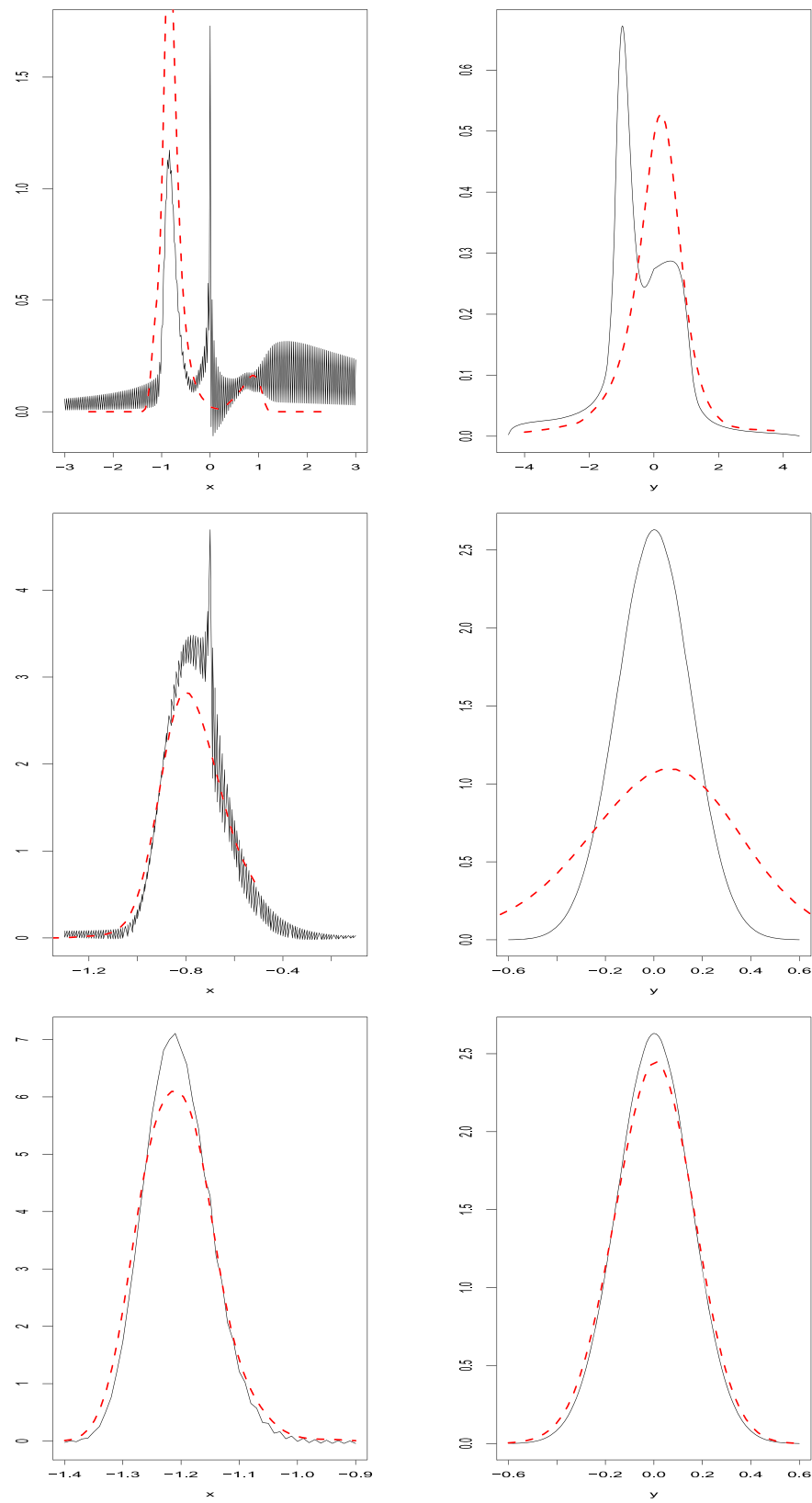

FIG. 3. Invariant density estimation. Left: marginal in $x$ of the estimation $\hat{p}_{\hat{b}}$ (red line) and true density approximated by a finite difference scheme (black line). Right: marginal in y of the estimation $\hat{p}_{\hat{b}}$ (red line) and true density approximated by a finite difference scheme (black line). Top line: set of parameters that generate spikes $(s=0, \beta=0.8, \tilde{\sigma}=0.3, \varepsilon=0.1$ and $\gamma=1.5)$. Middle line: set of parameters that generates few and small excursions $(s=0, \beta=0.8, \tilde{\sigma}=0.3, \varepsilon=0.4$ and $\gamma=1.5)$. Bottom line: set of parameters that does not generate spikes $(s=0, \beta=0.8, \tilde{\sigma}=0.3, \varepsilon=0.5$ and $\gamma=0.2)$. The finite difference scheme is unstable in the two first cases (black curve is very noisy). 

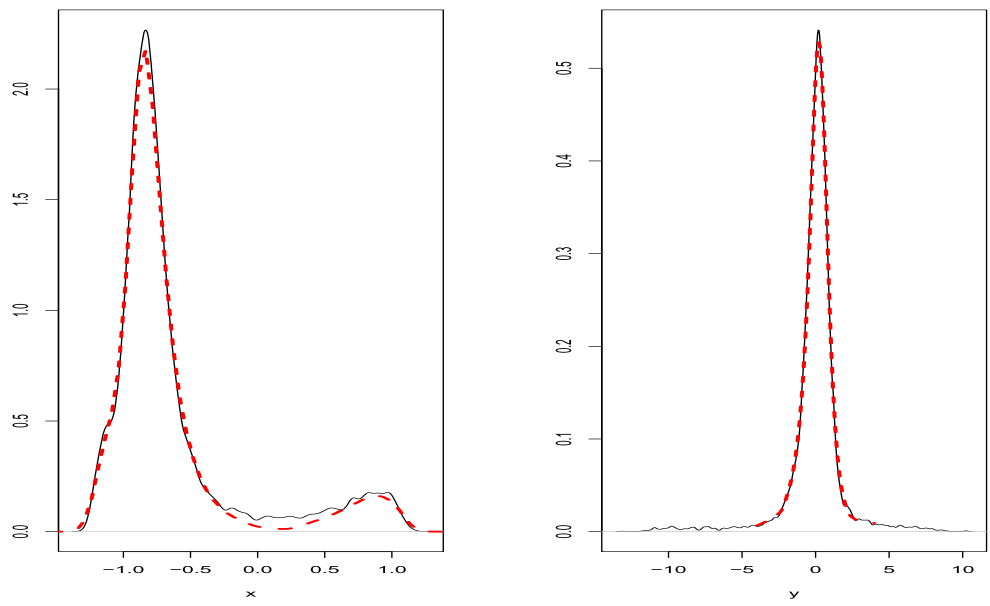

FIG. 4. Invariant density estimation. Left: marginal in $x$ of the estimation $\hat{p}_{\hat{\hat{b}}}$ (red line) and true density approximated by a Monte Carlo scheme (black line). Right: marginal in y of the estimation $\hat{p}_{\hat{b}}$ (red line) and true density approximated by a Monte Carlo scheme (black line). Simulations are performed with parameters that generate spikes $(s=0, \beta=0.8, \tilde{\sigma}=0.3, \varepsilon=0.1$ and $\gamma=1.5)$.

Then a kernel estimation procedure for i.i.d. data has been applied. The comparison with our estimation based on only one trajectory of dependent data is shown on Figure 4. The estimation is very close to the approximation of the stationary regime. Note also that in the spiking regime, as expected, the estimator of the marginal density in $x$ (red dotted line) has two bounds, one corresponding to the subthreshold activity and the other to the spiking activity. To conclude, our estimation procedure is stable whatever the value of $\varepsilon$ and fast to compute compared to the PDE solver or the Monte Carlo approximation.

Then the spike rate is estimated by formula (3.9) for the three sets of parameters that generate spikes, few spikes or no spikes. The expected number of up-crossings is estimated for level $u$ between -0.5 and 1.5. The three curves (as functions of $u$ ) are plotted in Figure 5: black plain line for the set of parameters that generate spikes $(s=0, \beta=0.8, \tilde{\sigma}=0.3, \varepsilon=0.1$ and $\gamma=1.5)$; red dotted line for the set of parameters that generates few and small excursions $(s=0, \beta=0.8, \tilde{\sigma}=0.3$, $\varepsilon=0.4$ and $\gamma=1.5)$; green dashed line for the set of parameters that does not generate spikes $(s=0, \beta=0.8, \tilde{\sigma}=0.3, \varepsilon=0.5$ and $\gamma=0.2)$. As expected, when spikes occur, the estimator is stable for $u \in[0 ; 0.8]$ (an interval that corresponds to the amplitude of large excursions) and then suddenly decreases to 0 . When no spike occur, the estimator is null. When only small sub-threshold excursions occur, the estimator decreases slowly to 0 .

We now focus on the two first cases (large or small excursions with $\varepsilon=0.1$ and $\varepsilon=0.4$, resp.). For both cases, we estimate the spike rate with the two approaches 


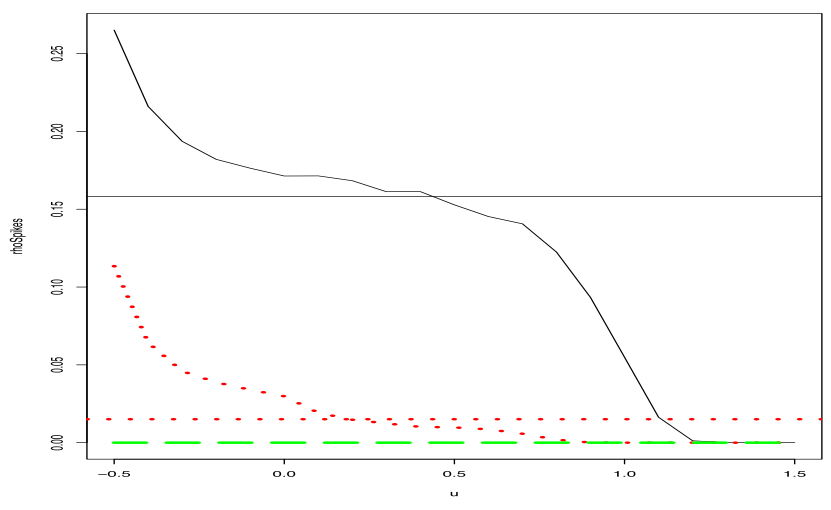

FIG. 5. Spike rate estimators $\hat{\lambda}(u)$ and $\hat{\rho}$ computed as the mean of $\rho(0.1), \ldots, \rho(0.6)$. Black plain curve: evolution of $\hat{\lambda}(u)$ with $u$ and black plain line $\hat{\rho}$ for a set of parameters that generate spikes $(s=0, \beta=0.8, \tilde{\sigma}=0.3, \varepsilon=0.1$ and $\gamma=1.5)$. Red dotted curve: evolution of $\hat{\lambda}(u)$ with $u$ and red dotted line $\hat{\rho}$ for a set of parameters that generates few and small excursions $(s=0, \beta=0.8$, $\tilde{\sigma}=0.3, \varepsilon=0.4$ and $\gamma=1.5)$. Green dashed curve: evolution of $\hat{\lambda}(u)$ with $u$ for a set of parameters that does not generate spikes ( $s=0, \beta=0.8, \tilde{\sigma}=0.3, \varepsilon=0.5$ and $\gamma=0.2$ ).

presented in the paper. First, we compute the mean of $\hat{\lambda}(u)$ for $u \in[0.1,0.6]$, this value is denoted $\bar{\lambda}$. Second, we compute $\rho$ defined as the number of spikes divided by the length of the time interval. As said before, spikes are defined as excursions in the phase space. Different thresholds $v \in[0.1,0.7]$ are used to define excursions $N_{t}(v)$. The mean of the corresponding spike rates is denoted $\bar{\rho}$ and superimposed on Figure 5 (horizontal lines). Table 1 displays the estimations $\bar{\rho}, \bar{\lambda}$, as well as the estimated mean and standard deviation [estimator (4.9)] of the length of intervals defined by two successive up-crossings. One can see that the two estimators $\bar{\lambda}$ and $\bar{\rho}$ are of the same order in both regimes. Therefore, the nonparametric estimation is a good alternative to the numerical approximation of the solution of the hypoelliptic Fokker-Planck equation, especially when the numerical scheme is not stable for $\varepsilon$ small. The up-crossing approach allows to estimate some characteristics of

TABLE 1

FitzHugh-Nagumo simulation for two regimes $(\varepsilon=0.1$ and $\varepsilon=0.4)$. Estimation of the spike rate by up-crossings approach $(\bar{\lambda})$ and by number of excursions $(\bar{\rho})$ and estimation of the mean and standard deviation of the length of intervals defined by two successive up-crossings

\begin{tabular}{cccc}
\hline Regime & $\overline{\boldsymbol{\rho}}$ & $\bar{\lambda}$ & $\begin{array}{c}\text { Length of intervals } \\
\text { Mean (sd) }\end{array}$ \\
\hline$\varepsilon=0.1$ & 0.1568 & 0.1609 & $6.35(6.32)$ \\
$\varepsilon=0.4$ & 0.0115 & 0.0111 & $93.13(82.70)$ \\
\hline
\end{tabular}


the length of the inter-up-crossings intervals (mean and standard deviation). In the spiking regime, mean and standard deviation are close. This is not the case when $\varepsilon$ increases.

6. Conclusion. The FitzHugh-Nagumo is a neuronal model that describes the generation of spikes at the intracellular level. In this paper, we study a stochastic version of the model from a probabilistic point of view. A transformed model is proposed that eases the study of hypoellipticity, as well as the existence and uniqueness of the stationary distribution. The bi-dimensional stochastic process is $\beta$-mixing. The stationary distribution can be estimated with an adaptive nonparametric estimator. Then we focus on the distribution of the length between successive spikes. We propose to study this distribution through the distribution of the number of up-crossings. The distribution function of the length of the interval between two successive up-crossings is defined through the stationary distribution. This allows to propose an estimator of the expectation of this distribution. We also derive the second moment of this distribution that allows to estimate the variance.

We illustrate the proposed estimators on a simulation study. Different regimes are explored, for different values of $\varepsilon$ : regime with no, few or high generation of spikes. The true stationary density has no explicit distribution. It can be approximated numerically by solving the Fokker-Planck equation. We consider a finite difference scheme, which is however unstable in the spiking regime. At the other hand, the nonparametric estimation of the stationary distribution reveals to be stable even in the spiking regime. We also implement the estimator of the mean length of the interval between two successive up-crossings. This estimator is based on the estimator of the stationary distribution. It reveals to be close to the mean spiking rate in the spiking regime.

It would be of interest in the future to apply the same approach to other stochastic intra-cellular neuronal models and to estimate the characteristics of their spiking process.

\section{APPENDIX: PROOF OF LEMMA 3.1}

Let $F \in B_{\Psi}$ and $\left\{F_{n}\right\}$ be a sequence of bounded functions such that $F_{n} \uparrow F$. Inequality (2.5) gives $\left\|P_{t} F\right\|_{\Psi} \leq\left(D \rho^{t}+1\right)\|F\|_{\Psi}<\infty$. Moreover, as in [26], let us introduce the exponential local martingale

$$
\begin{aligned}
M_{t}= & \exp \left(-\frac{1}{\sigma} \int_{0}^{t}\left(c\left(X_{s}, Y_{s}\right) Y_{s}+\nabla_{x} V\left(X_{s}\right)\right) d W_{s}\right. \\
& \left.+\frac{1}{2 \sigma^{2}} \int_{0}^{t}\left(c\left(X_{s}, Y_{s}\right) Y_{s}+\nabla_{x} V\left(X_{s}\right)\right)^{2} d s\right)
\end{aligned}
$$


By using monotone convergence theorem first and then the probabilistic representation as in [26], we get

$$
\begin{aligned}
\mathbb{E}^{\mathbb{P}_{z}^{0}\left[M_{t} F\left(\sigma \int_{0}^{t} W_{s} d s, \sigma W_{t}\right)\right]} & =\lim _{n \rightarrow \infty} \mathbb{E}^{\mathbb{P}_{z}^{0}}\left[M_{t} F_{n}\left(\sigma \int_{0}^{t} W_{s} d s, \sigma W_{t}\right)\right] \\
& =\lim _{n \rightarrow \infty} \int p_{t}\left(z, z^{\prime}\right) F_{n}\left(z^{\prime}\right) d z^{\prime} \\
& =\int p_{t}\left(z, z^{\prime}\right) F\left(z^{\prime}\right) d z^{\prime} \\
& =P_{t} F(z) \\
& <\infty .
\end{aligned}
$$

Then we recover the representation $P_{t} F(z)=\mathbb{E}^{\mathbb{P}_{z}^{0}}\left[M_{t} F\left(\sigma \int_{0}^{t} W_{s} d s, \sigma W_{t}\right)\right]$. Now function $G_{y_{1}}$ trivially belongs to $B_{\Psi}$. Hence, $P_{t} G_{y_{1}}(z)=\mathbb{E}^{\mathbb{P}_{z}^{0}}\left[M_{t}\left|\sigma W_{t}-y_{1}\right|\right]$. Thus by using the Lévy modulus of continuity of Brownian motion, we get

$$
\begin{aligned}
\left|P_{t} G_{y_{1}}(z)-\right| y-y_{1}|| & \leq \mathbb{E}^{\mathbb{P}_{z}^{0}}\left[M_{t}\left|\sigma W_{t}-y\right|\right] \\
& \leq C \sqrt{t \log \frac{1}{t}} \mathbb{E}^{z}\left[M_{t}\right] \\
& =O\left(t^{1 / 2-\varepsilon}\right)
\end{aligned}
$$

for $\varepsilon>0$. This proves the lemma. We remark that the convergence is uniform in $z$.

\section{REFERENCES}

[1] AzAïs, J.-M. and Wschebor, M. (2009). Level Sets and Extrema of Random Processes and Fields. Wiley, Hoboken, NJ. MR2478201

[2] Berglund, N. and Gentz, B. (2006). Noise-Induced Phenomena in Slow-Fast Dynamical Systems: A Sample-Paths Approach. Springer, London. MR2197663

[3] Berglund, N. and Landon, D. (2012). Mixed-mode oscillations and interspike interval statistics in the stochastic FitzHugh-Nagumo model. Nonlinearity 25.

[4] Cattiaux, P., Leon, J., Pineda Centeno, A. and Prieur, C. (2017). An overlook on statistical inference issues for stochastic damping Hamiltonian systems under the fluctuation-dissipation condition. Statistics 51 11-29.

[5] Comte, F., Prieur, C. and SAmson, A. (2017). Adaptive estimation for stochastic damping Hamiltonian systems under partial observation. Stochastic Process. Appl. 127 3689-3718. MR3707242

[6] Cramér, H. and Leadbetter, M. R. (1967). Stationary and Related Stochastic Processes. Wiley, New York.

[7] Ditlevsen, S. and Samson, A. (2017). Hypoelliptic diffusion: Discretization, filtering and inference from complete and partial observations. Submitted. 
[8] Di Bernardino, E., LeÓn, J. and Tchumatchenko, T. (2014). Cross-correlations and joint Gaussianity in multivariate level crossing models. J. Math. Neurosci. 4 Art. ID 22. MR3197020

[9] Doukhan, P. (1994). Mixing: Properties and Examples. Lecture Notes in Statistics 85. Springer, New York. MR1312160

[10] Gerstner, W. and Kistler, W. (2002). Spiking Neuron Models. Cambridge Univ. Press, Cambridge.

[11] Goldenshluger, A. and Lepski, O. (2011). Bandwidth selection in kernel density estimation: Oracle inequalities and adaptive minimax optimality. Ann. Statist. 39 1608-1632. MR2850214

[12] Hodgkin, A. L. and Huxley, A. F. (1952). A quantitative description of ion currents and its applications to conduction and excitation in nerve membranes. J. Physiol. 117 500-544.

[13] Konakov, V., Menozzi, S. and Molchanov, S. (2010). Explicit parametrix and local limit theorems for some degenerate diffusion processes. Ann. Inst. Henri Poincaré Probab. Stat. 46 908-923. MR2744877

[14] Kumar, P. and NARAYAnAn, S. (2006). Solution of Fokker-Planck equation by finite element and finite difference methods for nonlinear systems. Sädhanā 31 445-461.

[15] Lacour, C., Massart, P. and Rivoirard, V. (2017). Estimator selection: A new method with applications to kernel density estimation. Sankhya A 72 1-38. MR3707423

[16] Lindner, B., Garcia-Ojalvo, J., Neiman, A. and Schimansky-Geier, L. (2004). Effects of noise in excitable systems. Phys. Rep. 392 321-424.

[17] Lindner, B. and Schimansky-Geier, L. (1999). Analytical approach to the stochastic FitzHugh-Nagumo system and coherence resonance. Phys. Rev. E $607270-7276$.

[18] Longtin, A. (2000). Effect of noise on the tuning properties of excitable systems. Chaos Solitons Fractals 11 1835-1848.

[19] Mattingly, J. C., Stuart, A. M. and Higham, D. J. (2002). Ergodicity for SDEs and approximations: Locally Lipschitz vector fields and degenerate noise. Stochastic Process. Appl. 101 185-232. MR1931266

[20] MorRIS, C. and LECAR, H. (1981). Voltage oscillations in the barnacle giant muscle fiber. Biophys. J. 35 193-213.

[21] Pakdaman, K., Thieullen, M. and Wainrib, G. (2010). Fluid limit theorems for stochastic hybrid systems with application to neuron models. Adv. in Appl. Probab. 42 761-794. MR2779558

[22] Reynaud-Bouret, P., Rivoirard, V., Grammont, F. and Tuleau-Malot, C. (2014). Goodness-of-fit tests and nonparametric adaptive estimation for spike train analysis. J. Math. Neurosci. 4 Art. ID 3. MR3197017

[23] Reynaud-Bouret, P., Rivoirard, V. and Tuleau-Malot, C. (2013). Inference of functional connectivity in neurosciences via Hawkes processes. In 1st IEEE Global Conference on Signal and Information Processing, Austin, TX.

[24] Samson, A. and Thieullen, M. (2012). Contrast estimator for completely or partially observed hypoelliptic diffusion. Stochastic Process. Appl. 122 2521-2552. MR2926166

[25] Tuckwell, H. C. and Rodriguez, R. (1998). Analytical and simulation results for stochastic Fitzhugh-Nagumo neurons and neural networks. J. Comput. Neurosci. 5 91-113.

[26] WU, L. (2001). Large and moderate deviations and exponential convergence for stochastic damping Hamiltonian systems. Stochastic Process. Appl. 91 205-238. MR1807683 
InSTITUTO DE MATEMÁtica Y EstadísticA

RAFAEL LAGUARDIA

FACULTAD DE INGENIERÍA

UNIVERSIDAD DE LA REPÚBLICA

MONTEVIDEO

URUGUAY

AND

LABORATOIRE JEAN KUNTZMANN

UMR CNRS 5224

UNIVERSITÉ GRENOBLE ALPES

GRENOBLE

FRANCE

AND

INRIA PROJECT/TEAM AIRSEA

GRENOBLE

FRANCE

AND

EsCuela de Matemática

FACUlTAD DE CIENCIAS

Universidad CENTRAL DE VEnEZuela

CARACAS

VENEZUELA

E-MAIL: jose.leon@ciens.ucv.ve
LABORATOIRE JEAN KUNTZMANN

UMR CNRS 5224

UNIVERSITÉ GRENOBLE ALPES

GRENOBLE

FRANCE

E-MAIL: adeline.leclercq-samson@univ-grenoble-alpes.fr 